TRANSACTIONS OF THE

AMERICAN MATHEMATICAL SOCIETY

Volume 360, Number 8, August 2008, Pages 3975-4005

S 0002-9947(08)04396-1

Article electronically published on March 20, 2008

\title{
HOCHSCHILD COHOMOLOGY AND GRADED HECKE ALGEBRAS
}

\author{
ANNE V. SHEPLER AND SARAH WITHERSPOON
}

We dedicate this article to Sergey Yuzvinsky on the occasion of his 70th birthday.

\begin{abstract}
We develop and collect techniques for determining Hochschild cohomology of skew group algebras $S(V) \# G$ and apply our results to graded Hecke algebras. We discuss the explicit computation of certain types of invariants under centralizer subgroups, focusing on the infinite family of complex reflection groups $G(r, p, n)$ to illustrate our ideas. Resulting formulas for Hochschild two-cocycles give information about deformations of $S(V) \# G$ and, in particular, about graded Hecke algebras. We expand the definition of a graded Hecke algebra to allow a nonfaithful action of $G$ on $V$, and we show that there exist nontrivial graded Hecke algebras for $G(r, 1, n)$, in contrast to the case of the natural reflection representation. We prove that one of these graded Hecke algebras is equivalent to an algebra that has appeared before in a different form.
\end{abstract}

\section{INTRODUCTION}

Lusztig [18] showed that the graded version of an affine Hecke algebra essentially retains its representations, which in turn determine certain representations of the corresponding group of Lie type [15]. Lusztig's graded Hecke algebra for a Coxeter group is a deformation of the skew group algebra generated by the group and the vectors upon which the group acts (in its natural reflection representation). Drinfeld [9] had considered a different type of deformation, a degenerate affine Hecke algebra. He allowed any finite subgroup of $\mathrm{GL}(V)$ and used skew-symmetric forms on $V$ to deform the skew group algebra. Ram and the first author 22] showed that Lusztig's graded Hecke algebra was a special case of Drinfeld's construction. Drinfeld's algebra thus serves as a graded Hecke algebra for arbitrary finite subgroups of $\mathrm{GL}(V)$. One complication arises from this viewpoint when working with a wider class of groups: Drinfeld's construction for a given finite subgroup of $\mathrm{GL}(V)$ may only yield the trivial graded Hecke algebra. This article is partly motivated by a

Received by the editors January 20, 2006.

2000 Mathematics Subject Classification. Primary 16E40, 16 S80.

Key words and phrases. Graded Hecke algebra, degenerate affine Hecke algebra, deformation, Hochschild cohomology, reflection group, hyperplane arrangement, Ariki-Koike algebra.

The first author was partially supported by NSF grant \#DMS-0402819.

The second author was partially supported by NSF grant \#DMS-0443476 and the Alexander von Humboldt Foundation.

(C)2008 American Mathematical Society Reverts to public domain 28 years from publication 
desire to understand and resolve this problem. We use Hochschild cohomology as a tool to explore those deformations of skew group algebras that satisfy an expanded definition of graded Hecke algebra. To illustrate our ideas (and as an application of results), we focus on the infinite family of complex reflection groups.

Drinfeld's construction yields only the trivial algebra for the complex reflection groups $G(r, 1, n)=(\mathbb{Z} / r \mathbb{Z}) \prec \mathfrak{S}_{n}$ when $r \geq 3$ and $n \geq 4$ (see [22]). Ariki and Koike [1] defined a Hecke algebra (the Ariki-Koike algebra) for these complex reflection groups by generators and relations. Around the same time, Broué and Malle [2] defined a cyclotomic Hecke algebra for $G(r, 1, n)$ (coinciding with the Ariki-Koike algebra) and Broué, Malle, and Rouquier 4 gave a topological description using braid groups (see also [3]). Although the Hecke algebra itself enjoys fruitful study (see the survey paper by Mathas [20]), a satisfactory formulation of an affine Hecke algebra for the groups $G(r, 1, n)$ seems to be lacking. One asks when a graded Hecke algebra may be a reasonable substitute. In this article, we use Hochschild cohomology and algebraic deformation theory to address a natural question: When and what kinds of graded Hecke algebras may be defined for $G(r, 1, n)$ ?

Although Drinfeld's construction only yields trivial graded Hecke algebras for $G(r, 1, n)$ in general, Ram and the first author 22 constructed a novel graded Hecke algebra for these groups using ad hoc methods. Dezélée [7] independently discovered the same algebra as a subalgebra of a rational Cherednik algebra in the case $r=2$, that is, for the Weyl group $W B_{n}=G(2,1, n)$, and defined this algebra for $W D_{n}=G(2,2, n)$. Dezélée [8] further realized the graded Hecke algebras of Ram and the first author, for general $r$, as subalgebras of rational Cherednik algebras (equivalently, graded Hecke algebras associated to $V \oplus V^{*}$, where $V$ is the reflection representation). These rational Cherednik algebras are examples of symplectic reflection algebras, defined by Etingof and Ginzburg [11, who were interested in related deformations of orbifolds.

In this paper, we expand the definition of graded Hecke algebra to allow nonfaithful representations of a finite group $G$ on a finite dimensional vector space $V$ (Definition 8.1). We construct a natural graded Hecke algebra for $G(r, 1, n)$ using a nonfaithful representation, and we show that this construction coincides with the algebra of Ram and the first author 22] (Theorem 9.8). We further give the generic form of a graded Hecke algebra associated to $G(r, 1, n)$, depending on many parameters, in (9.1). A related paper of Chmutova [6] deals with existence of these more general types of graded Hecke algebras defined for any symplectic representation of $G$.

A graded Hecke algebra for a group $G$, with a given representation on a finite dimensional vector space $V$, is a particular type of deformation of the skew group algebra $S(V) \# G$. Thus every graded Hecke algebra defines an element in degree 2 Hochschild cohomology $\mathrm{HH}^{2}(S(V) \# G)$. We characterize all possible graded Hecke algebras, both in relation to Hochschild cohomology (Theorem 8.7) and in relation to defining skew-symmetric forms (Corollary 8.17). Corollary 8.17 generalizes a result of Ram and the first author 22, first formulated by Drinfeld [9] for Coxeter groups.

There may well be other compelling deformations of $S(V) \# G$ (arising from some of the many elements of degree 2 Hochschild cohomology) that are not graded Hecke algebras. Some small examples of such deformations were given in [5, 26. With these potential deformations in mind, as well as the immediate applications to 
graded Hecke algebras in this article, we compute the relevant cohomology in two cases:

(1) $G$ is the monomial reflection group $G(r, p, n)$ acting via its natural reflection representation on $V=\mathbb{C}^{n}$ (Theorem [5.1), and

(2) $G=G(r, p, n)$ acting on $V=\mathbb{C}^{n}$ via the permutation representation of its quotient symmetric group $\mathfrak{S}_{n}$ (Theorem 7.7).

The graded vector space structure of Hochschild cohomology $\mathrm{HH}^{*}(S(V) \# G)$ can be described in terms of invariants of centralizer subgroups of $G$ (see [12, 14]). We recall this structure in Section 3 and reformulate it in terms of semi-invariants. In Sections 5-7, we explicitly compute these semi-invariants in degree 2 using techniques of classical invariant theory and the theory of hyperplane arrangements; the full Hochschild cohomology space $\mathrm{HH}^{\circ}(S(V) \# G)$ could be computed similarly using our approach. Although we are primarily interested in applications to graded Hecke algebras and other deformations, our explicit computation of Hochschild cohomology should also be of interest to homological algebraists and to geometers studying cohomology for associated orbifolds.

Our results in case (1) above show that the lack of nontrivial graded Hecke algebras (when $r \geq 3, n \geq 4$, see 22 ) stems from a lack of certain types of elements in $\mathrm{HH}^{2}(S(V) \# G)$. This conclusion motivates our computation in case (2), leading to the aforementioned nontrivial graded Hecke algebras under a nonfaithful representation of $G$.

The authors thank A. Ram for questions and suggestions that led to this project.

\section{TERMinOLOGY AND NOTATION}

We work over the complex numbers $\mathbb{C}$, letting $\otimes=\otimes_{\mathbb{C}}$ unless otherwise specified. Let $G$ be a finite group and let $V$ be the vector space $\mathbb{C}^{n}$ with a representation of $G$. Let $V^{*}$ denote the contragredient (or dual) representation. We will often need a choice of basis $v_{1}, \ldots, v_{n}$ of $V$ and dual basis $x_{1}, \ldots, x_{n}$ of $V^{*}$.

Let $R$ be an associative $\mathbb{C}$-algebra with an action of $G$ by automorphisms. In this paper, $R$ will always be either the symmetric algebra $S(V)$ or the tensor algebra $T(V)$. The skew group algebra $R \# G$ is the vector space $R \otimes \mathbb{C} G$ with multiplication

$$
(r \otimes g)(s \otimes h)=r \cdot g(s) \otimes g h
$$

for all $r, s \in R$ and $g, h \in G$. We abbreviate $r \otimes g$ by $r \bar{g}(r \in R, g \in G)$ and $r \otimes 1$ or $1 \otimes g$ simply by $r$ or $\bar{g}$, respectively. Note that an element $g \in G$ acts on $R$ by conjugation by $\bar{g}: \bar{g} r(\bar{g})^{-1}=g(r) \bar{g}(\bar{g})^{-1}=g(r)$ for all $r \in R$.

For our cohomological computations, we fix some notation involving the action of $G$ on $V$. Let $V^{G}=\{v \in V: g(v)=v$ for all $g \in G\}$, the set of $G$-invariants in $V$. More generally, let $\chi: G \rightarrow \mathbb{C}$ be any linear character (i.e., any group homomorphism from $G$ to $\left.\mathbb{C}^{\times}\right)$and let $V^{\chi}=\{v \in V: g(v)=\chi(g) v$ for all $g \in G\}$, the set of $\chi$-invariants in $V$ (also called semi-invariants with respect to $\chi$ ).

For any $g \in G$, let $Z(g)=\{h \in G: g h=h g\}$ be the centralizer of $g$ in $G$, and let $V^{g}=\{v \in V: g(v)=v\}$ be the $g$-invariant subspace of $V$. Since $G$ is finite, we may assume $G$ acts by isometries on $V$ (i.e. $G$ preserves a Hermitian form). If $h \in Z(g)$, then $h$ preserves both $V^{g}$ and its orthogonal complement $\left(V^{g}\right)^{\perp}$ and we define

$$
h^{\perp}=\left.h\right|_{\left(V^{g}\right)^{\perp}}
$$


(i.e., $h^{\perp}$ is the linear transformation by which $h$ acts on the vector space $\left(V^{g}\right)^{\perp}$ ). We are particularly interested in semi-invariants with respect to the Hochschild character $\chi_{g}: Z(g) \rightarrow \mathbb{C}$ of $g$ defined by

$$
\chi_{g}(h)=\operatorname{det}\left(h^{\perp}\right)
$$

for all $h \in Z(g)$. When no confusion will arise, we simply write $\chi$ instead of $\chi_{g}$. Note that $\chi_{g}$ is a linear character. Let 1 denote the identity element of $G$ and let $I_{n}$ (or just $I$ ) denote the $n \times n$ identity matrix.

Our results involve the following complex reflection groups parametrized by positive integers $r, p, n$ : The full monomial group $G(r, 1, n)<\mathrm{GL}_{n}(\mathbb{C})$ is the group of all monomial $n \times n$ matrices whose nonzero entries are $r$-th roots of unity. The full monomial group is a finite complex reflection group acting on $V=\mathbb{C}^{n}$ and is isomorphic to the wreath product of the cyclic group of order $r$ and the symmetric group:

$$
G(r, 1, n)=N \cdot G(1,1, n) \cong(\mathbb{Z} / r \mathbb{Z})^{n} \rtimes \mathfrak{S}_{n}=\mathbb{Z} / r \mathbb{Z} \imath \mathfrak{S}_{n},
$$

where $N$ is the normal subgroup of diagonal matrices in $G(r, 1, n)$ and $G(1,1, n) \leq$ $G(r, 1, n)$ is isomorphic to the symmetric group $\mathfrak{S}_{n}$.

Let $\xi$ in $\mathbb{C}$ be a fixed primitive $r$-th root of unity. Let $\xi_{i}$ be the diagonal matrix $\operatorname{diag}\{1, \ldots, 1, \xi, 1, \ldots, 1\}$, with $i$-th entry $\xi$. We denote each element in $G(r, 1, n)$ by $g=\xi_{1}^{a_{1}} \ldots \xi_{n}^{a_{n}} \sigma$, where $\sigma \in \mathfrak{S}_{n}$ and $0 \leq a_{i}<r$. In the special case that $r$ is even (so $\xi^{r / 2}=-1$ ), we write $(1,-2):=\xi_{2}^{r / 2}(1,2)$ for the transformation $v_{2} \mapsto v_{1}$, $v_{1} \mapsto-v_{2}$.

For any $p$ dividing $r$, we consider a natural subgroup of $G(r, 1, n)$ also acting as a reflection group on $V$. Let $\psi(M)$ be the product of the nonzero entries of any matrix $M$. Then

$$
G(r, p, n)=\left\{M \in G(r, 1, n): \psi(M)^{r / p}=1\right\} ;
$$

that is, $M$ in $G(r, 1, n)$ lies in $G(r, p, n)$ when the sum of the exponents of $\xi$ in the nonzero entries of $M$ is 0 modulo $p$. Several real reflection groups (Coxeter groups) are special cases of the groups $G(r, p, n)$ :

- $G(1,1, n)$ is the symmetric group $\mathfrak{S}_{n}$,

- $G(2,1, n)$ is the Weyl group $W B_{n}$ of type $B_{n}$,

- $G(2,2, n)$ is the Weyl group $W D_{n}$ of type $D_{n}$, and

- $G(r, r, 2)$ is the dihedral group $I_{2}(r)$ of order $2 r$.

We briefly record some facts on centralizers and conjugacy classes in $G(r, 1, n)$. Note that $Z_{G(r, p, n)}(g)=Z_{G(r, 1, n)}(g) \cap G(r, p, n)$ for any $g$ in $G(r, p, n)$. The (generalized) cycle structure of an element $g$ in $G(r, 1, n)$ determines its conjugacy class and the size of its centralizer $Z(g)$ in the following way. Every element $g$ in $G(r, 1, n)$ can be written as the product of disjoint cycles of the form $\xi_{i}^{a_{i}} \xi_{i+1}^{a_{i+1}} \cdots \xi_{m}^{a_{m}}(i, i+1, \ldots, m)$. We call such an element an $(a, k)$-cycle, where $k=m-i+1$ and $a=a_{i}+a_{i+1}+\cdots+a_{m} \bmod r$. Two elements in $G(r, 1, n)$ are conjugate if and only if they have the same number of $(a, k)$-cycles for each $a$ and $k$ (where $0 \leq a<r$ and $1 \leq k \leq n$ ). Let $m_{a, k}$ be the number of $(a, k)$-cycles for $g$; the order of the centralizer of $g$ in $G(r, 1, n)$ is

$$
|Z(g)|=\prod_{a, k} m_{a, k} ! \cdot k^{m_{a, k}} \cdot r^{m_{a, k}} .
$$

See [22, Equation (2.5)] and [19, Appendix B, Section 3]. 


\section{Hochschild cohomology of $S(V) \# G$}

The Hochschild cohomology of a $\mathbb{C}$-algebra $R$ is the graded vector space $\mathrm{HH}^{*}(R)=\operatorname{Ext}_{R^{e}}(R, R)$, where $R^{e}=R \otimes R^{o p}$ acts on $R$ by left and right multiplication. More generally, if $M$ is an $R$-bimodule (equivalently a left $R^{e}$-module), then $\mathrm{HH}^{*}(R, M)=\operatorname{Ext}_{R^{e}}(R, M)$ so that $\mathrm{HH}^{*}(R)=\mathrm{HH}^{*}(R, R)$. For more details, see [25].

Fix a finite group $G$ and a representation $V=\mathbb{C}^{n}$ of $G$. The Hochschild cohomology of $S(V) \# G$ is given in [12, 14] when $G$ acts faithfully on $V$. In this section, we reformulate this result to aid our explicit computations and our determination of graded Hecke algebras. We state all results more generally for representations which are not necessarily faithful. No new techniques are needed for the generalization, and so we merely sketch those arguments that appear elsewhere for the faithful case.

Let $\mathcal{C}$ be a set of representatives of the conjugacy classes of $G$. In the following theorem, a negative exterior power is interpreted as 0. Recall the Hochschild characters $\chi_{g}$ defined in (2.1).

Theorem 3.1. There is an isomorphism of graded vector spaces

$$
\mathrm{HH}^{*}(S(V) \# G) \cong \bigoplus_{g \in \mathcal{C}}\left(S\left(V^{g}\right) \otimes \bigwedge^{\cdot-\operatorname{codim} V^{g}}\left(\left(V^{g}\right)^{*}\right)\right)^{\chi_{g}}
$$

For any $g \in \mathcal{C}$, let $\mathrm{HH}^{2}(g)$ be the $g$-component of $\operatorname{HH}^{2}(S(V) \# G)$ in the theorem:

$$
\mathrm{HH}^{2}(g)=\left(S\left(V^{g}\right) \otimes \bigwedge^{2-\operatorname{codim} V^{g}}\left(\left(V^{g}\right)^{*}\right)\right)^{\chi_{g}} .
$$

We call $\mathrm{HH}^{2}(g)$ the set of Hochschild semi-invariants of $g$. Note $\operatorname{HH}^{2}(g)=0$ if $\operatorname{codim} V^{g}>2$, since the exterior power $2-\operatorname{codim} V^{g}$ is negative in this case.

Sketch of a proof of Theorem 3.1. We give only an outline as the bulk of the calculation follows [12, 26]. A result of Ştefan on Hopf Galois extensions [24, Cor. 3.4] implies that there is an action of $G$ on $\mathrm{HH}^{\circ}(S(V), S(V) \# G)$ for which

$$
\mathrm{HH}^{*}(S(V) \# G) \cong \mathrm{HH}^{*}(S(V), S(V) \# G)^{G} .
$$

Now $S(V) \# G \cong \bigoplus_{g \in G} S(V) \bar{g}$ as an $S(V)$-bimodule. The above isomorphism of graded vector spaces may thus be rewritten as

$$
\mathrm{HH}^{*}(S(V) \# G) \cong\left(\bigoplus_{g \in G} \mathrm{HH}^{*}(S(V), S(V) \bar{g})\right)^{G} .
$$

The action of $G$ permutes the direct summands by conjugation on their indices $g \in G$. We may therefore rewrite the vector space structure of these $G$-invariants as $\bigoplus_{g \in \mathcal{C}} \mathrm{HH}^{*}(S(V), S(V) \bar{g})^{Z(g)}$ (one applies a transfer (trace) operator $\sum_{h \in G / Z(g)} h$ to each $Z(g)$-invariant to obtain a $G$-invariant). To compute the summands $\mathrm{HH}^{*}(S(V), S(V) \bar{g})^{Z(g)}$, one may first use a Koszul complex (see [25, §4.5] for details of Koszul complexes) to find $\mathrm{HH}^{*}(S(V), S(V) \bar{g})$ and then determine the $Z(g)$ invariants. The use of a Koszul complex yields

$$
\mathrm{HH}^{\cdot}(S(V) \# G) \cong \bigoplus_{g \in \mathcal{C}}\left(S\left(V^{g}\right) \bar{g} \otimes \bigwedge^{\cdot-\operatorname{codim} V^{g}}\left(\left(V^{g}\right)^{*}\right) \otimes \bigwedge^{\operatorname{codim} V^{g}}\left(\left(\left(V^{g}\right)^{\perp}\right)^{*}\right)\right)^{Z(g)},
$$


where $Z(g)$ acts diagonally on the given tensor product of three representations. Its action on the factor $S\left(V^{g}\right) \bar{g}$ is by conjugation by $\bar{h}(h \in Z(g))$. The actions on the given dual vector spaces are the contragredient actions. The factor $\bigwedge^{\operatorname{codim} V^{g}}\left(\left(\left(V^{g}\right)^{\perp}\right)^{*}\right)$ is one-dimensional since codim $V^{g}=\operatorname{dim}\left(\left(V^{g}\right)^{\perp}\right)^{*}$. We also denote this factor by $\operatorname{det}\left(\left(\left(V^{g}\right)^{\perp}\right)^{*}\right)$; it is included in the above expression (3.4) only since it carries a potentially nontrivial $Z(g)$-action.

We find the contribution to Hochschild cohomology from each $g \in G$ representing a conjugacy class by determining the $Z(g)$-invariants of

$$
S\left(V^{g}\right) \bar{g} \otimes \bigwedge^{\bullet-\operatorname{codim} V^{g}}\left(\left(V^{g}\right)^{*}\right) \otimes \operatorname{det}\left(\left(\left(V^{g}\right)^{\perp}\right)^{*}\right) .
$$

Let $x_{1}, \ldots, x_{m}$ be a basis of $\left(\left(V^{g}\right)^{\perp}\right)^{*}$. Let $s \in S\left(V^{g}\right) \otimes \bigwedge^{\bullet-\operatorname{codim} V^{g}}\left(\left(V^{g}\right)^{*}\right)$. Then the element $s \bar{g} \otimes x_{1} \wedge \cdots \wedge x_{m}$ of $S\left(V^{g}\right) \bar{g} \otimes \wedge^{\bullet-\operatorname{codim} V^{g}}\left(\left(V^{g}\right)^{*}\right) \otimes \operatorname{det}\left(\left(\left(V^{g}\right)^{\perp}\right)^{*}\right)$ is $Z(g)$-invariant if and only if

$$
s \bar{g} \otimes x_{1} \wedge \cdots \wedge x_{m}=\operatorname{det}\left(\left(h^{-1}\right)^{\perp}\right) h(s) \bar{g} \otimes x_{1} \wedge \cdots \wedge x_{m}
$$

for all $h \in Z(g)$. Equivalently, $h(s)=\operatorname{det}\left(h^{\perp}\right) s=\chi_{g}(h) s$ for all $h \in Z(g)$.

The next lemma gives in particular a necessary condition for $\operatorname{HH}^{2}(g)$ to be nonzero (cf. [12, Example 3.10]).

Lemma 3.6. Let $g \in G$, and suppose $\left(S\left(V^{g}\right) \otimes \bigwedge^{i-\operatorname{codim} V^{g}}\left(\left(V^{g}\right)^{*}\right)\right)^{\chi_{g}} \neq 0$ for some $i \geq 0$. If $h \in Z(g)$ and $\left.h\right|_{V^{g}}$ is the identity map, then $\operatorname{det}(h)=1$. In particular, $\operatorname{det}(g)=1$.

Proof. Let $s$ be a nonzero element of $\left(S\left(V^{g}\right) \otimes \bigwedge^{i-\operatorname{codim} V^{g}}\left(\left(V^{g}\right)^{*}\right)\right)^{\chi_{g}}$. If $h \in Z(g)$ and $\left.h\right|_{V^{g}}$ is the identity, then $h(s)=s$, and so $\operatorname{det}(h) s=\operatorname{det}\left(h^{\perp}\right) s=\chi_{g}(h) s=$ $h(s)=s$.

We may now rewrite Theorem 3.1 for degree 2 cohomology using (3.2) and the above lemma. The condition $\operatorname{det}(g)=1$ from the lemma implies $\operatorname{codim} V^{g} \neq 1$ if $\mathrm{HH}^{2}(g) \neq 0$, and the appearance of the exterior power $2-\operatorname{codim} V^{g}$ in (3.2) implies that $\operatorname{codim} V^{g} \leq 2$. Thus we have

$$
\mathrm{HH}^{2}(S(V) \# G) \cong \bigoplus_{g \in \mathcal{C}} \mathrm{HH}^{2}(g) \cong \bigoplus_{\begin{array}{c}
g \in \mathcal{C} \\
\operatorname{det}(g)=1 \\
\operatorname{codim} V^{g} \in\{0,2\}
\end{array}} \mathrm{HH}^{2}(g) .
$$

Remark 3.8. We briefly mention a generalization that we do not pursue in this paper. Let $\alpha: G \times G \rightarrow \mathbb{C}^{\times}$be a two-cocycle; that is, $\alpha(g, h) \alpha(g h, \ell)=\alpha(h, \ell) \alpha(g, h \ell)$ for all $g, h, \ell \in G$. The crossed product algebra $S(V) \#_{\alpha} G$ is the vector space $S(V) \otimes \mathbb{C} G$ with multiplication $(r \bar{g})(s \bar{h})=\alpha(g, h) r \cdot g(s) \overline{g h}$ for all $r, s \in R, g, h \in G$. The derivation of the more general Hochschild cohomology $\mathrm{HH}^{\bullet}\left(S(V) \#_{\alpha} G\right)$ is similar to that of $\mathrm{HH}^{\circ}(S(V) \# G)$. (See [26, Cor. 6.5], which remains valid for nonfaithful actions of $G$ on $V$.) One obtains a sum as in Theorem 3.1, namely

$$
\mathrm{HH}^{\bullet}\left(S(V) \#_{\alpha} G\right) \cong \bigoplus_{g \in \mathcal{C}}\left(S\left(V^{g}\right) \otimes \bigwedge^{\bullet-\operatorname{codim} V^{g}}\left(\left(V^{g}\right)^{*}\right)\right)^{\chi_{g}^{\alpha}} .
$$

However, the linear character $\chi_{g}^{\alpha}$ on $Z(g)$ now depends on $\alpha$ :

$$
\chi_{g}^{\alpha}(h)=\operatorname{det}\left(h^{\perp}\right) \alpha(g, h)(\alpha(h, g))^{-1}
$$


for all $h \in Z(g)$. These potentially different characters $\chi_{g}^{\alpha}$ can lead to different semi-invariants. In this broader setting, deformations of $S(V) \#_{\alpha} G$ are treated in [5, 6, 26, 27.

\section{INVARIANT THEORY OF $G(r, p, n)$}

The Hochschild cohomology of the skew group algebra $S(V) \# G(r, p, n)$ is derived from the invariant theory of $G(r, p, n)$ and its centralizer subgroups. In this section, we briefly record the invariant theory of $G(r, p, n)$ acting via its natural reflection representation on $V=\mathbb{C}^{n}$ and on the exterior algebra of derivations on $V^{*}$. We first recall some well-known facts on reflection groups.

Recall that a reflection is an element of $\operatorname{GL}(V)$ of finite order which fixes a hyperplane (called the reflecting hyperplane) in $V$ pointwise. Let $G$ be a reflection group, i.e., a (finite) group $G \leq \mathrm{GL}(V)$ generated by reflections. The collection of reflecting hyperplanes $\mathcal{A}$ for $G$ is called the reflection arrangement. For each $H \in \mathcal{A}$, let $l_{H}$ in $V^{*}$ be a linear form defining $H=\operatorname{ker} l_{H}$. The reflection arrangement $\mathcal{A}$ is defined (up to a nonzero constant) by the polynomial $Q=\prod_{H \in \mathcal{A}} l_{H}$ in $S\left(V^{*}\right)$. When $G$ is a reflection group acting on $V^{*}$, we identify $\left(V^{*}\right)^{*}$ with $V$ and define the reflection arrangement $\mathcal{A}$ by a polynomial $Q$ in $S(V)$.

Remark 4.1. By the Shephard-Todd-Chevalley Theorem (see [21, Chapter 6]), the invariant ring $S(V)^{G}$ is a polynomial algebra generated by $n$ algebraically independent invariants: $S(V)^{G}=\mathbb{C}\left[f_{1}, \ldots, f_{n}\right]$, for some polynomials $f_{i}$ called basic invariants. In fact, a set of homogeneous, algebraically independent, invariant polynomials $f_{1}, \ldots, f_{n}$ generate $S(V)^{G}$ if and only if the product of the degrees $\operatorname{deg} f_{i}$ is $|G|$ (for example, see [16, Theorem 3.7.5]). Hence, the invariants of a direct sum of reflection representations is the tensor product of the invariants: If $G$ and $G^{\prime}$ are reflection groups acting on complex vector spaces $V$ and $V^{\prime}$ with basic invariants $f_{1}, \ldots, f_{n}$ and $f_{1}^{\prime}, \ldots, f_{n^{\prime}}^{\prime}$, respectively, then

$$
S\left(V \oplus V^{\prime}\right)^{G \oplus G^{\prime}}=\mathbb{C}\left[f_{1}, \ldots, f_{n}, f_{1}^{\prime}, \ldots, f_{n^{\prime}}^{\prime}\right] \cong S(V)^{G} \otimes S\left(V^{\prime}\right)^{G^{\prime}} .
$$

The following proposition (see [23], for example, or above remark) describes the polynomials invariant under $G(r, p, n)$. Recall that the $k$-th elementary symmetric function in variables $z_{1}, \ldots, z_{n}$ is the polynomial $f_{k}=\sum_{1 \leq i_{1}<\cdots<i_{k} \leq n} z_{i_{1}} \cdots z_{i_{k}}$.

Proposition 4.2. Let $G=G(r, p, n)$ act on $V$ with $\mathbb{C}$-basis $v_{1}, \ldots, v_{n}$ via its natural reflection representation. Then

$$
S(V)^{G(r, p, n)}=\mathbb{C}\left[f_{1}, \ldots, f_{n}\right]
$$

where $f_{i}$ is the $i$-th elementary symmetric function in variables $v_{1}^{r}, \ldots, v_{n}^{r}$ for $i<n$ and $f_{n}=\left(v_{1} \cdots v_{n}\right)^{r / p}$.

We identify $S(V) \otimes \bigwedge^{k}\left(V^{*}\right)$ with the $S(V)$-module of $k$-derivations (or $k$-vector fields) on $V^{*}$. The following proposition (see [21]) describes the invariant derivations on $V^{*}$. The generators $\theta_{i}$ in the proposition are called basic derivations.

Proposition 4.3. Let $G$ be a reflection group acting on $V^{*}=\mathbb{C}^{n}$ with reflection arrangement defined by $Q \in S(V)$. Suppose $\theta_{1}, \ldots, \theta_{n}$ in $\left(S(V) \otimes V^{*}\right)^{G}$ are invariant derivations whose coefficient matrix has determinant $Q$ up to a nonzero scalar. Then

$$
\left(S(V) \otimes \bigwedge^{k}\left(V^{*}\right)\right)^{G}=\bigoplus_{1 \leq i_{1}<\cdots<i_{k} \leq n} S(V)^{G}\left(\theta_{i_{1}} \wedge \cdots \wedge \theta_{i_{k}}\right) .
$$


Proof. Note that $S(V)^{\operatorname{det}^{-1}}=S(V)^{G} Q$, where $\operatorname{det}^{-1}$ is the inverse of the determinant character of $G$ acting on $V^{*}$ (see [21, Example 6.40]). Solomon's Theorem [21, Prop. 6.47] then implies that the invariant $k$-derivations are generated freely over $S(V)^{G}$ by the nonzero wedge products of the $\theta_{i}$ (taken $k$ at a time).

We apply the above proposition to $G=G(r, p, n)$ (see [21, Appendix B]) .

Proposition 4.4. Let $G(r, p, n)$ act on $V=\mathbb{C}^{n}$ with $\mathbb{C}$-basis $v_{1}, \ldots, v_{n}$ and on $V^{*}$ contragrediently with dual basis $x_{1}, \ldots, x_{n}$. Then

$$
\left(S(V) \otimes \bigwedge\left(V^{*}\right)\right)^{G(r, p, n)}=\bigoplus_{1 \leq k \leq n} \bigoplus_{1 \leq i_{1}<\cdots<i_{k} \leq n} S(V)^{G(r, p, n)}\left(\theta_{i_{1}} \wedge \cdots \wedge \theta_{i_{k}}\right),
$$

where

$$
\theta_{j}:=\sum_{1 \leq i \leq n} v_{i}^{(j-1) r+1} \otimes x_{i}
$$

for $j<n$ and

$$
\theta_{n}:= \begin{cases}\sum_{1 \leq i \leq n} v_{i}^{(n-1) r+1} \otimes x_{i} & \text { if } p \neq r \\ \sum_{1 \leq i \leq n}\left(v_{1} \cdots v_{i-1} v_{i+1} \cdots v_{n}\right)^{r-1} \otimes x_{i} & \text { if } p=r .\end{cases}
$$

\section{Hochschild COHOMOlOGy IN DEgRee 2 For $G(r, p, n)$}

We give the Hochschild 2-cohomology $\mathrm{HH}^{2}(S(V) \# G)$ explicitly when the monomial group $G=G(r, p, n)$ acts (faithfully) via its natural reflection representation on $V=\mathbb{C}^{n}$. The results rely on some computations postponed until Section 6. We assume $n \geq 4$ throughout this section. Results for $n=2$ and $n=3$ are slightly different because the pattern of relevant conjugacy classes in $G$ differs for low values of $n$. The techniques from this section apply to give explicit Hochshild cohomology in the case $n=2$ or $n=3$, but we omit these results for the sake of brevity. In the following theorem, we use the notation $\mathrm{HH}^{2}(g)$ defined in (3.2).

Theorem 5.1. Assume $n \geq 4$. Let $G=G(r, p, n)$ act on $V=\mathbb{C}^{n}$ via the natural reflection representation. The Hochschild cohomology in degree 2 for the skew group algebra $S(V) \# G$ is

$$
\mathrm{HH}^{2}(S(V) \# G) \cong \mathrm{HH}^{2}(1) \oplus \mathrm{HH}^{2}(1,2,3) \oplus \mathrm{HH}^{2}(1,-2) \oplus \bigoplus_{i=1, \ldots,\lfloor r / 2\rfloor} \mathrm{HH}^{2}\left(\xi_{1}^{i} \xi_{2}^{-i}\right)
$$

where

- $\mathrm{HH}^{2}(1)=\left(S(V) \otimes \bigwedge^{2}\left(V^{*}\right)\right)^{G(r, p, n)}$ is given explicitly in Proposition 4.4,

- $\operatorname{HH}^{2}(1,2,3)$ is given explicitly in Proposition 6.1 .

- $\mathrm{HH}^{2}(1,-2)=0$ unless $r=2 p$, in which case $\operatorname{HH}^{2}(1,-2)$ is given explicitly in Proposition 6.4.

- $\mathrm{HH}^{2}\left(\xi_{1}^{\ell} \xi_{2}^{-\ell}\right)=0$ unless $r>1, p=r$, and $\ell \neq r / 2$, in which case $\mathrm{HH}^{2}\left(\xi_{1}^{\ell} \xi_{2}^{-\ell}\right)$ is given explicitly in Proposition 6.10.

Proof. We apply (3.7). If $g \in G(r, p, n)$ with $\operatorname{codim}\left(V^{g}\right)=0$, then $g$ is the identity element 1 (as $G(r, p, n)$ acts faithfully on $V$ ). Furthermore, the Hochschild character $\chi$ for $g=1$ should be interpreted as the trivial character of $Z(g)=G(r, p, n)$ since 
$\left(V^{g}\right)^{\perp}=\{0\}$. Hence

$$
\mathrm{HH}^{2}(1)=\left(S\left(V^{g}\right) \otimes \bigwedge^{2}\left(\left(V^{g}\right)^{*}\right)\right)^{G(r, p, n)},
$$

which is given explicitly in Proposition 4.4 .

If $g \in G(r, p, n)$ with $\operatorname{codim}\left(V^{g}\right)=2$, then $g$ is conjugate to one of the following elements (see [22, Section 2B]):

$$
\begin{array}{lll}
g_{b}=\xi_{1}^{a} \xi_{3}^{-a}(1,2,3), & 0 \leq a \leq \operatorname{gcd}(p, 3)-1, \\
g_{c}=\xi_{1}^{a+\ell} \xi_{2}^{-a}(1,2), & & \ell \neq 0(\text { so } r \neq 1), \\
g_{d}=\xi_{1}^{\ell_{1}} \xi_{2}^{\ell_{2}}, & & \ell_{1} \neq 0, \ell_{2} \neq 0(\text { so } r \neq 1), \\
g_{e}=(1,2) \xi_{3}^{\ell}, & & \ell \neq 0, \\
g_{f}=(1,2) \xi_{3}^{a} \xi_{4}^{-a}(3,4) . &
\end{array}
$$

We can further conjugate these elements into simpler forms under our assumption $n \geq 4$. Each element of the form $g_{b}=\xi_{1}^{a} \xi_{3}^{-a}(1,2,3)$ is conjugate to $(1,2,3)$ (via $\left.(1,2,3) \xi_{3}^{a} \xi_{4}^{-a}\right)$ in $G(r, p, n)$. Each element of the form $g_{c}=\xi_{1}^{a+\ell} \xi_{2}^{-a}(1,2)$ is conjugate to $\xi_{2}^{\ell}(1,2)$ (via $\xi_{1}^{a}(1,2) \xi_{3}^{-a}$ ) in $G(r, p, n)$. We assume $1=\operatorname{det} g_{c}=-\xi^{\ell}$, else $\mathrm{HH}^{2}\left(g_{c}\right)=0$ by Lemma 3.6. Thus, if $\mathrm{HH}\left(g_{c}\right)$ is nonzero, $r$ must be even with $\xi^{\ell}=-1$ and we may assume $g_{c}=\xi_{2}^{\ell}(1,2)=(1,-2)$. The sets of Hochschild semi-invariants $\mathrm{HH}^{2}(1,2,3)$ and $\mathrm{HH}^{2}(1,-2)$ are given in Propositions 6.1 and 6.4 .

By Proposition 6.8, the set of Hochschild semi-invariants $\operatorname{HH}^{2}\left(g_{d}\right)$ is zero unless $G=G(r, r, n)$. The element $g_{d}$ lies in $G(r, r, n)$ only when $\ell_{2} \equiv-\ell_{1} \bmod r$. In the special case that $\ell_{1}=\ell_{2}=r / 2, \mathrm{HH}^{2}\left(g_{d}\right)$ is zero by Proposition 6.9. Otherwise, $\mathrm{HH}^{2}\left(g_{d}\right)$ is given in Proposition 6.10.

The sets of Hochschild semi-invariants $\mathrm{HH}^{2}\left(g_{e}\right)$ and $\mathrm{HH}^{2}\left(g_{f}\right)$ are both zero by Propositions 6.11 and 6.12.

The Hochschild cohomology in degree 2 simplifies when $G$ is the symmetric group $\mathfrak{S}_{n}=G(1,1, n)$ and when $G$ is the Weyl group $W B_{n}=G(2,1, n)$. (See Proposition 4.2 for the explicit rings of invariants $S(V)^{\mathfrak{S}_{n}}$ and $S(V)^{\mathrm{WB}_{n}}$ needed in the following statements.)

Corollary 5.2. Let $G$ be the symmetric group $\mathfrak{S}_{n}=G(1,1, n)$ for $n \geq 4$ acting on $V$ with $\mathbb{C}$-basis $v_{1}, \ldots, v_{n}$ (via the natural permutation representation). The Hochschild cohomology in degree 2 for the skew group algebra $S(V) \# G$ is

$$
\mathrm{HH}^{2}(S(V) \# G) \cong \mathrm{HH}^{2}(1) \oplus \mathrm{HH}^{2}(1,2,3)
$$

where

- $\mathrm{HH}^{2}(1)=\left(S(V) \otimes \bigwedge^{2}\left(V^{*}\right)\right)^{\mathfrak{S}_{n}}=\bigoplus_{1 \leq i_{1}<i_{2} \leq n} S(V)^{\mathfrak{S}_{n}}\left(\theta_{i_{1}} \wedge \theta_{i_{2}}\right)$, for

$$
\theta_{j}:=\sum_{1 \leq i \leq n} v_{i}^{j-1} \otimes x_{i},
$$

- $\operatorname{HH}^{2}(1,2,3)=\mathbb{C}\left[f_{0}, f_{1}, \ldots, f_{n-3}\right]$, where $f_{0}=\left(v_{1}+v_{2}+v_{3}\right)$ and $f_{i}$ is the $i$-th elementary symmetric function in $v_{4}, \ldots, v_{n}$ for $i>0$. 
Corollary 5.3. Let $G$ be the Weyl group $W B_{n}=G(2,1, n)$ for $n \geq 4$ acting on $V$ with $\mathbb{C}$-basis $v_{1}, \ldots, v_{n}$ (via its natural reflection representation). The Hochschild cohomology in degree 2 for the skew group algebra $S(V) \# G$ is

$$
\mathrm{HH}^{2}(S(V) \# G) \cong \operatorname{HH}^{2}(1) \oplus \mathrm{HH}^{2}(1,2,3) \oplus \mathrm{HH}^{2}(1,-2)
$$

where

- $\mathrm{HH}^{2}(1)=\left(S(V) \otimes \bigwedge^{2}\left(V^{*}\right)\right)^{W B_{n}}=\bigoplus_{1 \leq i_{1}<i_{2} \leq n} S(V)^{W B_{n}}\left(\theta_{i_{1}} \wedge \theta_{i_{2}}\right)$, for

$$
\theta_{j}:=\sum_{1 \leq i \leq n}\left(v_{i}\right)^{2(j-1)+1} \otimes x_{i},
$$

- $\operatorname{HH}^{2}(1,2,3)=\mathbb{C}\left[f_{0}, f_{1}, \ldots, f_{n-3}\right]$, where $f_{0}=\left(v_{1}+v_{2}+v_{3}\right)^{2}$ and $f_{i}$ is the $i$-th elementary symmetric function in $v_{4}^{2}, \ldots, v_{n}^{2}$ for $i>0$,

- $\operatorname{HH}^{2}(1,-2)=\mathbb{C}\left[f_{1}^{\prime}, \ldots, f_{n-2}^{\prime}\right]$, where $f_{i}^{\prime}$ is the $i$-th elementary symmetric function in $v_{3}^{2}, \ldots, v_{n}^{2}$.

\section{INVARIANT THEORY FOR CERTAIN CENTRALIZER SUBGROUPS OF $G(r, p, n)$}

In Section [5, we gave the Hochschild cohomology of $S(V) \# G$ in degree 2, where $V$ is the natural reflection representation of $G=G(r, p, n)$. The Hochschild cohomology can be expressed as the direct sum of certain subspaces, each subspace invariant under an action of a centralizer subgroup $Z(g)$ for some $g \in G$. In this section, we determine these invariant subspaces. We analyze the contribution $\mathrm{HH}^{2}(g)$ from the group elements $g \in G(r, p, n)$ with $\operatorname{codim}\left(V^{g}\right)=2$ and $\operatorname{det} g=1$. Recall that $v_{1}, \ldots, v_{n}$ is the given $\mathbb{C}$-basis for $V$.

Proposition 6.1. Assume $n \geq 4$ and $G=G(r, p, n)$. Let $g=(1,2,3)$. Then

$$
S\left(V^{g}\right)^{Z(g)}=\bigoplus_{\substack{0 \leq i<r, 0 \leq j<m \\ i \equiv 3 j r / p} \bmod r} \mathbb{C}\left[f_{0}^{r}, f_{1}, \ldots, f_{n^{\prime}-1}, f_{n^{\prime}}^{m}\right] \quad f_{0}^{i} f_{n^{\prime}}^{j},
$$

where

- $n^{\prime}=n-3$,

- $m=p / 3$ if 3 divides $p ; m=p$ otherwise,

- $f_{0}=v_{1}+v_{2}+v_{3}$,

- $f_{n^{\prime}}=\left(v_{4} \cdots v_{n}\right)^{r / p}$,

- $f_{i}$ is the $i$-th elementary symmetric function in $v_{4}^{r}, \ldots, v_{n}^{r}$ for $1 \leq i \leq n^{\prime}-1$.

Let $\chi$ be the Hochschild character for $g$ as defined in (2.1). The set of Hochschild semi-invariants for $g$ is

$$
\mathrm{HH}^{2}(g)=S\left(V^{g}\right)^{\chi}=\bigoplus_{\substack{0 \leq i<r, 0 \leq j<m \\ i \equiv 2+3 j r / p \bmod r}} \mathbb{C}\left[f_{0}^{r}, f_{1}, \ldots, f_{n^{\prime}-1}, f_{n^{\prime}}^{m}\right] \quad f_{0}^{i} f_{n^{\prime}}^{j} .
$$

Proof. Let $v_{0}=f_{0}=v_{1}+v_{2}+v_{3}$. Note that $V^{g}=\mathbb{C}$-span $\left\{v_{0}, v_{4}, \ldots, v_{n}\right\}$ and $\left(V^{g}\right)^{\perp} \subset \mathbb{C}-\operatorname{span}\left\{v_{1}, v_{2}, v_{3}\right\}$. Let $V_{A}=\mathbb{C}-\operatorname{span}\left\{v_{1}, v_{2}, v_{3}\right\}$ and $V_{B}=\mathbb{C}-\operatorname{span}\left\{v_{4}, \ldots\right.$, $\left.v_{n}\right\}$. One may use (2.2) to verify that the centralizer of $g$ in $G$ is

$$
Z(g)=\left\{h_{A} \oplus h_{B}: h_{A} \in\left\langle\xi I_{3},(1,2,3)\right\rangle<G(r, 1,3), h_{B} \in G\left(r, 1, n^{\prime}\right)\right\} \cap G(r, p, n) .
$$


The centralizer $Z(g)$ includes the subgroup

$$
Z^{\prime}=\left\{I_{3} \oplus h_{B}: h_{B} \in G\left(r, p, n^{\prime}\right)\right\} .
$$

Since $\chi(h)=1$ for all $h \in Z^{\prime}, S\left(V^{g}\right)^{\chi} \subset S\left(V^{g}\right)^{Z^{\prime}}$, and since $Z^{\prime} \subset Z(g)$, we have $S\left(V^{g}\right)^{Z(g)} \subset S\left(V^{g}\right)^{Z^{\prime}}$. We begin by finding the invariants of $S\left(V^{g}\right)$ under $Z^{\prime}$.

The group $Z^{\prime}$ acts on $V_{B}$ as the reflection group $G\left(r, p, n^{\prime}\right)$ with invariants

$$
S\left(V_{B}\right)^{G\left(r, p, n^{\prime}\right)}=\mathbb{C}\left[v_{4}, \ldots, v_{n}\right]^{G\left(r, p, n^{\prime}\right)}=\mathbb{C}\left[f_{1}, \ldots, f_{n^{\prime}}\right]
$$

by Proposition 4.2. The group $Z^{\prime}$ fixes $v_{0}$, so acts on $V^{g}$ as the direct sum of the trivial group with $G\left(r, p, n^{\prime}\right)$. Thus, $S\left(V^{g}\right)^{Z^{\prime}}=\mathbb{C}\left[v_{0}, v_{4}, \ldots, v_{n}\right]^{Z^{\prime}}=\mathbb{C}\left[f_{0}, f_{1}, \ldots, f_{n^{\prime}}\right]$ by Remark 4.1 . Hence,

$$
S\left(V^{g}\right)^{Z(g)} \subset \mathbb{C}\left[f_{0}, f_{1}, \ldots, f_{n^{\prime}}\right] \text { and } S\left(V^{g}\right)^{\chi} \subset \mathbb{C}\left[f_{0}, f_{1}, \ldots, f_{n^{\prime}}\right] .
$$

The polynomials $f_{1}, \ldots, f_{n^{\prime}-1}$ are invariant under $Z(g)$. But the polynomials $f_{0}$ and $f_{n^{\prime}}$ pick up a scalar when $Z(g)$ acts. We introduce some characters to record and compare the actions of $Z(g)$ on $f_{0}$ and on $f_{n^{\prime}}$.

Define a linear character on $Z(g)$,

$$
\tau: Z(g) \rightarrow \mathbb{C},
$$

by $h\left(f_{0}\right)=\tau(h) f_{0}$ for all $h$ in $Z(g)$. Fix $h=h_{A} \oplus h_{B} \in Z(g)$ and note that $h_{A}=\left(\xi^{k} I_{3}\right)(1,2,3)^{j}$, for some $j$ and $k$. Since $\operatorname{det}\left(h^{\perp}\right)=\xi^{2 k}\left(\right.$ as $\left.\left(\operatorname{dim} V^{g}\right)^{\perp}=2\right)$, $\tau^{2}(h)=\xi^{2 k}=\operatorname{det}\left(h^{\perp}\right)=\chi(h)$. Hence, $\chi=\tau^{2}$.

Recall that $\psi(M)$ is the product of the nonzero entries of $M$, for any matrix $M$. Since $M \in G(r, p, n)$ implies that $\psi(M)^{r / p}=1$, we see that $\psi\left(h_{B}\right)^{r / p}=\psi\left(h_{A}\right)^{-r / p}$ $\left(\right.$ as $\left.\psi(h)=\psi\left(h_{A}\right) \psi\left(h_{B}\right)\right)$. Observe that $\psi\left(h_{A}\right)=\xi^{3 k}=\tau(h)^{3}$. Then

$$
h\left(f_{n^{\prime}}\right)=\left[\psi\left(h_{B}\right)\left(v_{4} \cdots v_{n}\right)\right]^{r / p}=\psi\left(h_{B}\right)^{r / p} f_{n^{\prime}}=\psi\left(h_{A}\right)^{-r / p} f_{n^{\prime}}=\tau(h)^{-3 r / p} f_{n^{\prime}} .
$$

In summary, the action of $Z(g)$ on $f_{0}, \ldots, f_{n}^{\prime}$ is given by

$$
h\left(f_{k}\right)=\left\{\begin{aligned}
\tau(h) f_{k} & \text { for } k=0, \\
f_{k} & \text { for } 0<k<n^{\prime}, \\
\tau(h)^{-3 r / p} f_{k} & \text { for } k=n^{\prime}
\end{aligned}\right.
$$

for all $h \in Z(g)$. In particular, for any $i, j$,

$$
\begin{aligned}
h\left(f_{0}^{i} f_{n^{\prime}}^{j}\right) & =\tau(h)^{i-3 j r / p} f_{0}^{i} f_{n^{\prime}}^{j}, \quad \text { and } \\
\chi(h) & =\tau^{2}(h) .
\end{aligned}
$$

We now give an explicit description of $S\left(V^{g}\right)^{Z(g)}$ and $S\left(V^{g}\right)^{\chi}$ as subsets of $\mathbb{C}\left[f_{0}, \ldots, f_{n^{\prime}}\right]$. Fix some $s$ in $\mathbb{C}\left[f_{0}, \ldots, f_{n^{\prime}}\right]$ and write $s$ as a polynomial in $f_{0}$ and $f_{n^{\prime}}$ with coefficients in $f_{1}, \ldots, f_{n^{\prime}-1}$ :

$$
s=\sum_{i, j \geq 0} a_{i j} f_{0}^{i} f_{n^{\prime}}^{j}, \quad \text { where } a_{i j} \in \mathbb{C}\left[f_{1}, \ldots, f_{n^{\prime}-1}\right] \subset S\left(V^{g}\right)^{Z(g)} .
$$

Then $s$ is invariant (respectively $\chi$-invariant) under $Z(g)$ exactly when each $f_{0}^{i} f_{n^{\prime}}^{j}$ with $a_{i j} \neq 0$ is invariant (respectively $\chi$-invariant) under $Z(g)$. The monomial $f_{0}^{i} f_{n^{\prime}}^{j}$ is invariant under $Z(g)$ exactly when

$$
\tau(h)^{i-3 j r / p}=1 \quad \text { for all } h \in Z(g)
$$


by Equation (6.3). Similarly, the monomial $f_{0}^{i} f_{n^{\prime}}^{j}$ is $\chi$-invariant under $Z(g)$ exactly when

$$
\begin{array}{rlrl}
\tau(h)^{2} f_{0}^{i} f_{n^{\prime}}^{j}=\chi(h) f_{0}^{i} f_{n^{\prime}}^{j} & =h\left(f_{0}^{i} f_{n^{\prime}}^{j}\right)=\tau(h)^{i-3 j r / p} f_{0}^{i} f_{n^{\prime}}^{j}, & \text { i.e., } \\
\tau(h)^{2+3 j r / p-i} & =1 \quad \text { for all } h \in Z(g) .
\end{array}
$$

But the set $\{\tau(h): h \in Z(g)\}$ depends on $p$ and $n$.

Note that $\tau(h)$ is a power of $\xi$ for each $h \in Z(g)$ and that $h^{\prime}=\xi_{1} \xi_{2} \xi_{3} \xi_{4}^{-3} \in Z(g)$ with $\tau\left(h^{\prime}\right)=\xi$. Hence

$$
\{\tau(h): h \in Z(g)\}=\langle\xi\rangle
$$

Thus the monomial $f_{0}^{i} f_{n^{\prime}}^{j}$ is invariant under $Z(g)$ exactly when $\xi^{i-3 j r / p}=1$ :

$$
f_{0}^{i} f_{n^{\prime}}^{j} \in S\left(V^{g}\right)^{Z(g)} \quad \text { if and only if } \quad i \equiv 3 j r / p \bmod r .
$$

In particular, $f_{0}^{r}$ is invariant under $Z(g)$. Also, $f_{n^{\prime}}^{m}$ is invariant under $Z(g)$ where $m=p / 3$ if $p$ is divisible by 3 and $m=p$ otherwise. So

$$
\mathbb{C}\left[f_{0}^{r}, f_{1}, \ldots, f_{n^{\prime}-1}, f_{n^{\prime}}^{m}\right] \subset S\left(V^{g}\right)^{Z(g)} \subset \mathbb{C}\left[f_{0}, f_{1}, \ldots, f_{n^{\prime}-1}, f_{n^{\prime}}\right] .
$$

Thus, $S\left(V^{g}\right)^{Z(g)}$ is the free $\mathbb{C}\left[f_{0}^{r}, f_{1}, \ldots, f_{n^{\prime}-1}, f_{n^{\prime}}^{m}\right]$-module

$$
S\left(V^{g}\right)^{Z(g)}=\bigoplus_{\substack{0 \leq i<r, 0 \leq j<m \\ i \equiv 3 j r / p \bmod r}} \mathbb{C}\left[f_{0}^{r}, f_{1}, \ldots, f_{n^{\prime}-1}, f_{n^{\prime}}^{m}\right] \quad f_{0}^{i} f_{n^{\prime}}^{j} .
$$

Similarly, $f_{0}^{i} f_{n^{\prime}}^{j}$ is $\chi$-invariant under $Z(g)$ exactly when $\xi^{2+3 j r / p-i}=1$ :

$$
f_{0}^{i} f_{n^{\prime}}^{j} \in S\left(V^{g}\right)^{\chi} \quad \text { if and only if } \quad i \equiv 2+3 j r / p \bmod r .
$$

Thus, $S\left(V^{g}\right)^{\chi}$ is the free $\mathbb{C}\left[f_{0}^{r}, f_{1}, \ldots, f_{n^{\prime}-1}, f_{n^{\prime}}^{m}\right]$-module

$$
S\left(V^{g}\right)^{\chi}=\bigoplus_{\substack{0 \leq i<r, 0 \leq j<m \\ i \equiv 2+3 j r / p} \bmod r} \mathbb{C}\left[f_{0}^{r}, f_{1}, \ldots, f_{n^{\prime}-1}, f_{n^{\prime}}^{m}\right] \quad f_{0}^{i} f_{n^{\prime}}^{j}
$$

Proposition 6.4. Assume $n \geq 4, G=G(r, p, n)$. Suppose $g=(1,-2)$. Then

$$
S\left(V^{g}\right)^{Z(g)}= \begin{cases}\mathbb{C}\left[f_{1}, \ldots, f_{n^{\prime}-1}, f_{n^{\prime}}^{p / 2}\right] & \text { if } p \text { is even, } \\ \mathbb{C}\left[f_{1}, \ldots, f_{n^{\prime}-1}, f_{n^{\prime}}^{p}\right] & \text { if } p \text { is odd, }\end{cases}
$$

where

- $n^{\prime}=n-2$,

- $f_{n^{\prime}}=\left(v_{3} \ldots v_{n}\right)^{r / p}$,

- $f_{i}$ is the $i$-th elementary symmetric function in $v_{3}^{r}, \ldots, v_{n}^{r}$ for $i<n^{\prime}$.

Let $\chi$ be the Hochschild character for $g$ as defined in (2.1). The set of Hochschild semi-invariants for $g$ is

$$
\mathrm{HH}^{2}(g)=S\left(V^{g}\right)^{\chi}=\bigoplus_{\substack{0 \leq i<r \\ 2 \equiv 2(-r / p) i} \bmod r} \mathbb{C}\left[f_{1}, \ldots, f_{n^{\prime}-1}, f_{n^{\prime}}^{r}\right] \quad f_{n^{\prime}}^{i} .
$$

In particular, $\mathrm{HH}^{2}(g)$ is zero unless $r=2 p$. 
Proof. Each element $h$ of the centralizer $Z(g)$ breaks into a direct sum: We may write $h=h^{\perp} \oplus h_{\perp}$, where $h^{\perp} \in G(r, 1,2)$ is the matrix of $h$ acting on $\left(V^{g}\right)^{\perp}$ (with respect to the basis $\left\{v_{1}, v_{2}\right\}$ of $\left.\left(V^{g}\right)^{\perp}\right)$ and $h_{\perp} \in G\left(r, 1, n^{\prime}\right)$ is the matrix of $h$ acting on $V^{g}$ (with respect to the basis $\left\{v_{3}, \ldots, v_{n}\right\}$ of $V^{g}$ ). One may use (2.2) to verify that

$$
Z(g)=\left\{h^{\perp} \oplus h_{\perp}: h^{\perp} \in\left\langle g^{\perp}, \xi I_{2}\right\rangle, h_{\perp} \in G\left(r, 1, n^{\prime}\right)\right\} \cap G(r, p, n) .
$$

The centralizer $Z(g)$ includes the subgroup $Z^{\prime}$ of $G$ fixing $\left(V^{g}\right)^{\perp}$ pointwise:

$$
Z^{\prime}=\left\{I_{2} \oplus B: B \in G\left(r, p, n^{\prime}\right)\right\} \subset Z(g) .
$$

The group $Z^{\prime}$ acts on $V^{g}=\mathbb{C}$-span $\left\{v_{3}, \ldots, v_{n}\right\}$ as the reflection group $G\left(r, p, n^{\prime}\right)$. By Proposition 4.2 .

$$
S\left(V^{g}\right)^{Z^{\prime}}=\mathbb{C}\left[v_{3}, \ldots, v_{n}\right]^{G\left(r, p, n^{\prime}\right)}=\mathbb{C}\left[f_{1}, \ldots, f_{n^{\prime}}\right] .
$$

Since $\chi(h)=1$ for all $h \in Z^{\prime}, S\left(V^{g}\right)^{\chi} \subset S\left(V^{g}\right)^{Z^{\prime}}$, and since $Z^{\prime} \subset Z(g), S\left(V^{g}\right)^{Z(g)}$ $\subset S\left(V^{g}\right)^{Z^{\prime}}$. Hence,

$$
S\left(V^{g}\right)^{Z(g)} \subset \mathbb{C}\left[f_{1}, \ldots, f_{n^{\prime}}\right] \text { and } S\left(V^{g}\right)^{\chi} \subset \mathbb{C}\left[f_{1}, \ldots, f_{n^{\prime}}\right] .
$$

Observe that $f_{1}, \ldots, f_{n^{\prime}-1}$ lie in $S\left(V^{g}\right)^{Z(g)}$. How does $Z(g)$ act on the last polynomial, $f_{n^{\prime}}$ ? Recall that $\psi(M)$ is the product of the nonzero entries of $M$, for any matrix $M$, and that $M \in G(r, p, n)$ implies $\psi(M)^{r / p}=1$. For any $h \in Z(g)$, $\psi(h)=\psi\left(h^{\perp}\right) \psi\left(h_{\perp}\right)$ and

$$
\begin{aligned}
h\left(f_{n^{\prime}}\right) & =h\left(v_{3} \cdots v_{n}\right)^{r / p} \\
& =\left(\psi\left(h_{\perp}\right) v_{3} \cdots v_{n}\right)^{r / p} \\
& =\psi\left(h_{\perp}\right)^{r / p} f_{n^{\prime}} \\
& =\psi(h)^{r / p} \psi\left(h^{\perp}\right)^{-r / p} f_{n^{\prime}} \\
& =\psi\left(h^{\perp}\right)^{-r / p} f_{n^{\prime}} .
\end{aligned}
$$

Fix some $s$ in $\mathbb{C}\left[f_{1}, \ldots, f_{n^{\prime}}\right]$ and write $s$ as a polynomial in $f_{n^{\prime}}$ with coefficients in $f_{1}, \ldots, f_{n^{\prime}-1}$ :

$$
s=\sum_{i \geq 0} a_{i} f_{n^{\prime}}^{i} \quad \text { where } \quad a_{i} \in \mathbb{C}\left[f_{1}, \ldots, f_{n^{\prime}-1}\right] \subset S\left(V^{g}\right)^{Z(g)} .
$$

Then $s$ is invariant (respectively $\chi$-invariant) under $Z(g)$ exactly when each $f_{n^{\prime}}^{i}$ with $a_{i} \neq 0$ is invariant (respectively $\chi$-invariant) under $Z(g)$.

From Equation (6.5), the polynomial $f_{n^{\prime}}^{i}$ is invariant under $Z(g)$ exactly when

$$
1=\psi\left(h^{\perp}\right)^{-i r / p} \quad \text { for all } h \in Z(g) .
$$

Also, the polynomial $f_{n^{\prime}}^{i}$ is $\chi$-invariant under $Z(g)$ exactly when

$$
\begin{aligned}
\operatorname{det}\left(h^{\perp}\right) f_{n^{\prime}}^{i} & =\chi(h) f_{n^{\prime}}^{i}=h\left(f_{n^{\prime}}^{i}\right)=\psi\left(h^{\perp}\right)^{-i r / p} f_{n^{\prime}}^{i}, \quad \text { i.e., } \\
\operatorname{det}\left(h^{\perp}\right) & =\psi\left(h^{\perp}\right)^{-i r / p}, \quad \text { for all } h \in Z(g) .
\end{aligned}
$$

Note that $Z(g)^{\perp}:=\left\{h^{\perp}: h \in Z(g)\right\}$ is generated by $g^{\perp}$ and $\xi I_{2}=\xi_{1} \xi_{2}$ since $n \geq 3$ implies that the centralizer $Z(g)$ contains $\xi_{1} \xi_{2} \xi_{3}^{-2} \in G(r, p, n)$. (If $n=2$, the matrix $\xi I_{2}$ need not lie in $G(r, p, n)$.) Thus, in order to establish the invariance or semi-invariance of $f_{n^{\prime}}^{i}$, we need only check Equations (6.6) and (6.7) for $g^{\perp}$ and $\xi I_{2}$. Note that $g=\xi_{2}^{r / 2}(1,2) \in G(r, p, n)$ implies that the integer $r / p$ is even. 
Hence $\psi\left(g^{\perp}\right)^{-i r / p}=(-1)^{-i r / p}=1$, and $\operatorname{det}\left(g^{\perp}\right)=1$. Hence, both Equations (6.6) and (6.7) are satisfied for the generator $g^{\perp}$ of $Z(g)^{\perp}$. So, the invariance or semiinvariance of $f_{n^{\prime}}^{i}$ depends only on $i$ and the action of the generator $\xi I_{2}$ of $Z(g)^{\perp}$ on $f_{n^{\prime}}$.

Thus, $f_{n^{\prime}}^{i}$ is invariant under $Z(g)$ exactly when

$$
1=\psi\left(\xi I_{2}\right)^{-i r / p}=\left(\xi^{2}\right)^{-i r / p}, \quad \text { i.e. } \quad 0 \equiv 2 i r / p \quad \bmod r .
$$

But $0 \equiv 2 i r / p \bmod r$ means that $i$ is a multiple of $p / 2$ when $p$ is even and a multiple of $p$ when $p$ is odd. Also, $f_{n^{\prime}}^{i}$ is $\chi$-invariant under $Z(g)$ exactly when

$$
\xi^{2}=\operatorname{det}\left(\xi I_{2}\right)=\psi\left(\xi I_{2}\right)^{-i r / p}=\left(\xi^{2}\right)^{-i r / p}, \quad \text { i.e. } \quad 2 \equiv-2 i r / p \quad \bmod r .
$$

In particular, $f_{n^{\prime}}^{r}$ is invariant under $Z(g)$. Hence

$$
S\left(V^{g}\right)^{Z(g)}= \begin{cases}\mathbb{C}\left[f_{1}, \ldots, f_{n^{\prime}-1}, f_{n^{\prime}}^{p / 2}\right] & \text { if } p \text { is even, } \\ \mathbb{C}\left[f_{1}, \ldots, f_{n^{\prime}-1}, f_{n^{\prime}}^{p}\right] & \text { if } p \text { is odd, }\end{cases}
$$

and

$$
\mathrm{HH}^{2}(g)=S\left(V^{g}\right)^{\chi}=\bigoplus_{\substack{0 \leq i<r \\ 2 \equiv 2(-r / p) i} \bmod r} \mathbb{C}\left[f_{1}, \ldots, f_{n^{\prime}-1}, f_{n^{\prime}}^{r}\right] \quad f_{n^{\prime}}^{i} .
$$

Proposition 6.8. Assume $n \geq 2, r \geq 2, G=G(r, p, n)$. Let $g=\xi_{1}^{\ell_{1}} \xi_{2}^{\ell_{2}}$, where $\ell_{i} \neq 0$. The set of Hochschild semi-invariants for $g$ is trivial,

$$
\mathrm{HH}^{2}(g)=S\left(V^{g}\right)^{\chi}=0,
$$

unless $G=G(r, r, n)$.

Proof. Note that $V^{g}=\mathbb{C}-\operatorname{span}\left\{v_{3}, \ldots, v_{n}\right\}$ and $\left(V^{g}\right)^{\perp}=\mathbb{C}$-span $\left\{v_{1}, v_{2}\right\}$. Let $h=$ $\xi_{1} \xi_{2}^{p-1} \in Z(g)$. Then $\left.h\right|_{V^{g}}=1$ and $\operatorname{det} h^{\perp}=\xi^{p}$. Lemma 3.6 implies that if $\mathrm{HH}^{2}(g)$ is nonzero, then $\xi^{p}=1$ and so $p=r$.

Proposition 6.9. Assume $n \geq 2, r \geq 2$ ( $r$ even). Let $G=G(r, r, n)$. Let $g=$ $\xi_{1}^{r / 2} \xi_{2}^{r / 2}$. The set of Hochschild semi-invariants for $g$ is trivial:

$$
\mathrm{HH}^{2}(g)=S\left(V^{g}\right)^{\chi}=0 .
$$

Proof. Note that $V^{g}=\mathbb{C}$-span $\left\{v_{3}, \ldots, v_{n}\right\}$ and $\left(V^{g}\right)^{\perp}=\mathbb{C}$-span $\left\{v_{1}, v_{2}\right\}$. Let $h=$ $(1,2)$. Then $h \in Z(g)$ with $\left.h\right|_{V^{g}}=1$. But $\operatorname{det} h^{\perp}=-1 \neq 1$. By Lemma 3.6. $\mathrm{HH}^{2}(g)$ is zero.

Proposition 6.10. Assume $n \geq 3, r \geq 2$. Let $G=G(r, r, n)$. Let $g=\xi_{1}^{\ell} \xi_{2}^{-\ell}$, where $\ell \neq r / 2$. Then

$$
S\left(V^{g}\right)^{Z(g)}=\mathbb{C}\left[f_{1}, \ldots, f_{n^{\prime}-1}, f_{n^{\prime}}^{r}\right]
$$

where

- $n^{\prime}=n-2$,

- $f_{n^{\prime}}=v_{3} \cdots v_{n}$,

- $f_{i}$ is the $i$-th elementary symmetric function in $v_{3}^{r}, \ldots, v_{n}^{r}$ for $i<n^{\prime}$.

Let $\chi$ be the Hochschild character for $g$ as defined in (2.1). The set of Hochschild semi-invariants for $g$ is

$$
\mathrm{HH}^{2}(g)=S\left(V^{g}\right)^{\chi}=\mathbb{C}\left[f_{1}, \ldots, f_{n^{\prime}-1}, f_{n^{\prime}}^{r}\right] \quad f_{n^{\prime}}^{r-1} .
$$


Proof. Note that $V^{g}=\mathbb{C}$-span $\left\{v_{3}, \ldots, v_{n}\right\}$ and $\left(V^{g}\right)^{\perp}=\mathbb{C}$-span $\left\{v_{1}, v_{2}\right\}$. Let $s \in$ $\mathrm{HH}^{2}(g)$ be a Hochschild semi-invariant for $g$. Consider the subgroup of $Z(g)$,

$$
Z^{\prime}=\left\{I_{2} \oplus B: B \in G\left(r, r, n^{\prime}\right)\right\} .
$$

Since $\operatorname{det} h^{\perp}=1$ for all $h \in Z^{\prime}, s$ is invariant under $Z^{\prime}$, that is, $s \in S\left(V^{g}\right)^{Z^{\prime}}=$ $\mathbb{C}\left[v_{3}, \ldots, v_{n}\right]^{Z^{\prime}}$. But $Z^{\prime}$ acts on the vector space $V^{g}$ as $G\left(r, r, n^{\prime}\right)$ with respect to the basis $\left\{v_{3}, \ldots, v_{n}\right\}$ of $V^{g}$. By Proposition 4.2

$$
S\left(V^{g}\right)^{Z^{\prime}}=\mathbb{C}\left[v_{3}, \ldots, v_{n}\right]^{G\left(r, r, n^{\prime}\right)}=\mathbb{C}\left[f_{1}, \ldots, f_{n^{\prime}}\right] .
$$

Hence,

$$
\mathrm{HH}^{2}(g)=\{\text { Hochschild semi-invariants for } g\} \subset \mathbb{C}\left[f_{1}, \ldots, f_{n^{\prime}}\right] .
$$

One may use (2.2) to verify that

$$
Z(g)=\{A \oplus B \in G(r, r, n): A \text { is a } 2 \times 2 \text { diagonal matrix }\} .
$$

Let $s$ be any element of $\mathbb{C}\left[f_{1}, \ldots, f_{n^{\prime}}\right]$ and write $s$ as a polynomial in $f_{n^{\prime}}$ with coefficients in $f_{1}, \ldots, f_{n^{\prime}-1}$ :

$$
s=\sum_{i \geq 0} a_{i} f_{n^{\prime}}^{i} \quad \text { where each } a_{i} \in \mathbb{C}\left[f_{1}, \ldots, f_{n^{\prime}-1}\right] .
$$

Note that $f_{1}, \ldots, f_{n^{\prime}-1}$ are invariant under $Z(g)$. Hence $s$ is invariant (respectively $\chi$-invariant) under $Z(g)$ exactly when each $f_{n^{\prime}}^{i}$ with $a_{i} \neq 0$ is invariant (respectively $\chi$-invariant) under $Z(g)$.

Observe that

$$
h\left(f_{n^{\prime}}\right)=\chi(h)^{-1} f_{n^{\prime}}=\left(\operatorname{det} h^{\perp}\right)^{-1} f_{n^{\prime}}
$$

for any $h \in Z(g)$ since $p=r$. Hence, $f_{n^{\prime}}^{i}$ is invariant under $Z(g)$ exactly when

$$
1=\left(\operatorname{det} h^{\perp}\right)^{-i} \quad \text { for all } h \in Z(g) .
$$

Similarly, $f_{n^{\prime}}$ is $\chi$-invariant under $Z(g)$ exactly when

$$
\begin{array}{rlrl}
\operatorname{det}\left(h^{\perp}\right) & =\left(\operatorname{det} h^{\perp}\right)^{-i} & \text { for all } h \in Z(g), & \text { i.e., } \\
1 & =\left(\operatorname{det} h^{\perp}\right)^{1+i} \quad & \text { for all } h \in Z(g) .
\end{array}
$$

As $n \geq 3,\left\{\operatorname{det} h^{\perp}: h \in Z(g)\right\}=\langle\xi\rangle$. Hence, $f_{n^{\prime}}^{i}$ is invariant under $Z(g)$ exactly when $i$ is a multiple of $r$, and $f_{n^{\prime}}^{i}$ is $\chi$-invariant under $Z(g)$ exactly when $-1 \equiv i$ $\bmod r$. Thus,

$$
S\left(V^{g}\right)^{Z(g)}=\mathbb{C}\left[f_{1}, \ldots, f_{n^{\prime}-1}, f_{n^{\prime}}^{r}\right]
$$

and

$$
\mathrm{HH}^{2}(g)=S\left(V^{g}\right)^{\chi}=\mathbb{C}\left[f_{1}, \ldots, f_{n^{\prime}-1}, f_{n^{\prime}}^{r}\right] \quad f_{n^{\prime}}^{r-1} .
$$

Proposition 6.11. Assume $r \geq 2, G=G(r, p, n)$. Let $g=(1,2) \xi_{3}^{\ell}$. The set of Hochschild semi-invariants for $g$ is zero:

$$
\mathrm{HH}^{2}(g)=S\left(V^{g}\right)^{\chi}=0 .
$$

Proof. Note $V^{g}=\mathbb{C}$-span $\left\{v_{1}+v_{2}, v_{4}, v_{5}, \ldots, v_{n}\right\}$ and $\left(V^{g}\right)^{\perp}=\mathbb{C}$-span $\left\{v_{1}-v_{2}, v_{3}\right\}$. Let $h=(1,2) \in Z(g)$. Then $\left.h\right|_{V^{g}}=1$ while $\operatorname{det} h^{\perp}=-1$, so Lemma 3.6 implies that $\mathrm{HH}^{2}(g)=0$. 
Proposition 6.12. Assume $n \geq 4$. Let $g=(1,2) \xi_{3}^{a} \xi_{4}^{-a}(3,4)$. The set of Hochschild semi-invariants for $g$ is zero:

$$
\mathrm{HH}^{2}(g)=S\left(V^{g}\right)^{\chi}=0 .
$$

Proof. Note that $V^{g}=\mathbb{C}$-span $\left\{v_{1}+v_{2}, v_{3}+\xi^{-a} v_{4}, v_{5}, \ldots, v_{n}\right\}$ and that $\left(V^{g}\right)^{\perp}=$ $\mathbb{C}$-span $\left\{v_{2}-v_{1}, v_{3}-\xi^{-a} v_{4}\right\}$. Let $h=(1,2) \in Z(g)$. Then $\left.h\right|_{V^{g}}=1$ while $\operatorname{det} h^{\perp}=$ -1 , so Lemma 3.6 implies that $\operatorname{HH}^{2}(g)=0$.

\section{HochSCHILD 2-COHOMOLOGY FOR NONFAITHFul ACTION OF $G(r, p, n)$}

Let $G=G(r, 1, n)$, with $n \geq 3$. We define a nonfaithful action of $G$ on $V=\mathbb{C}^{n}$ and determine the Hochschild 2-cohomology for the skew group algebra $S(V) \# G$. This nonfaithful action will yield nontrivial graded Hecke algebras in Section 9 .

Although we restrict our attention to $G(r, 1, n)$ in this section, the same results generally hold for $G(r, p, n)$ (under the nonfaithful action). In fact, Propositions 7.3 and 7.6 and Theorem 7.7 below hold verbatim when we replace $G(r, 1, n)$ by $G(r, p, n)$ and replace $n \geq 3$ by $n \geq 5$. We simply intersect each centralizer $Z(g)$ in $G(r, 1, n)$ with $G(r, p, n)$ throughout the proofs. We leave the details to the reader. (For $p \geq 2$ and low values of $n$, Remark 7.4 does not apply as the centralizers exhibit a different structure.)

Recall that $G$ is the group product $N \cdot G(1,1, n)$, where $N$ is the normal subgroup of diagonal matrices in $G$ and $G(1,1, n) \leq G$ is isomorphic to $\mathfrak{S}_{n}$. Define a representation

$$
\rho: G \rightarrow \mathrm{GL}(V)
$$

by composing the quotient map $G \rightarrow \mathfrak{S}_{n}$ with the permutation representation of $\mathfrak{S}_{n}$ on $V$ (permuting the fixed basis $v_{1}, \ldots, v_{n}$ ).

The next lemma describes the centralizer of a diagonal element. Given a diagonal matrix $g=\xi^{a_{1}} I_{n_{1}} \oplus \cdots \oplus \xi^{a_{k}} I_{n_{k}}$, rename the basis vectors $v_{1}, \ldots, v_{n}$ of $V$ so that $v_{1}^{(1)}, \ldots, v_{n_{1}}^{(1)}$ are the first $n_{1}$ basis vectors, $v_{1}^{(2)}, \ldots, v_{n_{2}}^{(2)}$ are the next $n_{2}$ basis vectors, etc., and decompose $V$ as $V_{1} \oplus \cdots \oplus V_{k}$ with $V_{i}=\mathbb{C}$-span $\left\{v_{1}^{(i)}, \ldots, v_{n_{i}}^{(i)}\right\}$. One may use (2.2) to verify the following lemma.

Lemma 7.2. Let $g^{\prime}$ be a diagonal matrix in $G=G(r, 1, n)$ and suppose $n_{1}, \ldots, n_{k}$ are the multiplicities of the diagonal entries. Then $g^{\prime}$ is conjugate in $G$ to some $g=\xi^{a_{1}} I_{n_{1}} \oplus \cdots \oplus \xi^{a_{k}} I_{n_{k}}$ and $Z(g)$ acts on $V=V_{1} \oplus \cdots \oplus V_{k}$ as the direct sum of reflection groups,

$$
Z(g)=\left\{M_{1} \oplus \cdots \oplus M_{k}: M_{i} \in G\left(r, 1, n_{i}\right)\right\}=G\left(r, 1, n_{1}\right) \oplus \cdots \oplus G\left(r, 1, n_{k}\right) .
$$

Contribution from $g$ with $\operatorname{codim} V^{g}=0$.

We first find the contribution to the Hochschild 2-cohomology of $S(V) \# G(r, 1, n)$ from $g$ with $\operatorname{codim} V^{g}=0$. The set of $g$ in $G=G(r, 1, n)$ with $\operatorname{codim}\left(V^{g}\right)=0$ under the nonfaithful representation $\rho$ is exactly the subgroup $N$ of diagonal matrices in $G(r, 1, n)$. Below, we use the notation $\operatorname{HH}^{2}(g)$ defined in (3.2) and we identity $S(V) \otimes \wedge^{k}\left(V^{*}\right)$ with the $S(V)$-module of $k$-derivations on $V^{*}$.

Proposition 7.3. Let $G=G(r, 1, n)$ act on $V=\mathbb{C}^{n}$ via the nonfaithful representation $\rho$. Let $g^{\prime} \in G$ be a diagonal matrix. Then $g^{\prime}$ is conjugate to some $g \in G$ whose set of Hochschild semi-invariants $\mathrm{HH}^{2}(g)$ is the set of derivation 2-forms invariant under a direct sum of symmetric groups. 
Explicitly: Suppose $n_{1}, \ldots, n_{k}$ are the multiplicities of the diagonal entries of $g^{\prime}$ and decompose $V$ as $V_{1} \oplus \cdots \oplus V_{k}$ with each $V_{i} \cong \mathbb{C}^{n_{i}}$ as above. For $i=1, \ldots, k$, let $\theta_{1}^{(i)}, \ldots, \theta_{n_{i}}^{(i)} \in S\left(V_{i}\right) \otimes V_{i}^{*}$ be basic derivations for the permutation action of the symmetric group $\mathfrak{S}_{n_{i}}$ on $V_{i}$. Then $\mathrm{HH}^{2}(g)$ is generated by the wedge products of the $\theta_{j}^{(i)}$ taken two at a time:

$$
\mathrm{HH}^{2}(g)=\left(S(V) \otimes \bigwedge^{2}\left(V^{*}\right)\right)^{Z(g)}=\bigoplus_{\substack{i \leq i^{\prime}, j<j^{\prime} \text { when } i=i^{\prime}}} S(V)^{Z(g)}\left(\theta_{j}^{(i)} \wedge \theta_{j^{\prime}}^{\left(i^{\prime}\right)}\right) .
$$

Also,

$$
S(V)^{Z(g)}=\bigoplus_{i=1}^{k} S\left(V_{i}\right)^{\mathfrak{S}_{n_{i}}}
$$

Remark 7.4. We may easily construct explicit basic invariants and basic derivations for the last proposition using Propositions 4.2 and 4.4. Let $x_{1}^{(i)}, \ldots, x_{n_{i}}^{(i)}$ be the basis of $V_{i}^{*}$ dual to the basis $v_{1}^{(i)}, \ldots, v_{n_{i}}^{(i)}$ of $V_{i}$. Let $f_{j}^{(i)}$ be the $j$-th elementary symmetric function in $v_{1}^{(i)}, \ldots, v_{n_{i}}^{(i)}$. Then

$$
S(V)^{Z(g)}=\mathbb{C}\left[f_{1}^{(1)}, \ldots, f_{n_{1}}^{(1)}, \ldots, f_{1}^{(k)}, \ldots, f_{n_{k}}^{(k)}\right] .
$$

We may also explicitly define $\theta_{1}^{(i)}, \ldots, \theta_{n_{i}}^{(i)} \in S\left(V_{i}\right) \otimes V_{i}^{*}$ :

$$
\theta_{j}^{(i)}:=\sum_{1 \leq l \leq n_{i}}\left(v_{l}^{(i)}\right)^{(j-1) r+1} \otimes x_{l}^{(i)} .
$$

Proof of Proposition 7.3. By Lemma 7.2, $g^{\prime}$ is conjugate to some diagonal $g$ with

$$
Z(g)=G\left(r, 1, n_{1}\right) \oplus \cdots \oplus G\left(r, 1, n_{k}\right) .
$$

Under the representation $\rho, g$ acts as the identity and $V^{g}=V$. Thus, the Hochschild character $\chi: Z(g) \rightarrow \mathbb{C}$ (given by $h \mapsto \operatorname{det} h^{\perp}$ ) is trivial and $\operatorname{HH}^{2}(g)$ is just the set of derivation 2 -forms invariant under $Z(g)$,

$$
\mathrm{HH}^{2}(g)=\left(S\left(V^{g}\right) \otimes \bigwedge^{2-\operatorname{codim} V^{g}}\left(\left(V^{g}\right)^{*}\right)\right)^{\chi}=\left(S(V) \otimes \bigwedge^{2}\left(V^{*}\right)\right)^{Z(g)} .
$$

Each $G\left(r, 1, n_{i}\right)$ acts on $V_{i}$ as the symmetric group $\mathfrak{S}_{n_{i}} \cong G\left(1,1, n_{i}\right)$ under the representation $\rho$. Thus the block diagonal group $Z(g)$ acts as the direct sum of symmetric groups, and we simply find the polynomials and derivation 2-forms invariant under the permutation action of $\mathfrak{S}_{n_{1}} \oplus \cdots \oplus \mathfrak{S}_{n_{k}}$ on $V_{1} \oplus \cdots \oplus V_{k}$.

By Remark 4.1, the ring of invariant polynomials is just the tensor product of the corresponding rings $S\left(V_{i}\right)^{\mathfrak{S}_{n_{i}}}$ of symmetric polynomials. The underlying arrangement of reflecting hyperplanes is given by the product of the polynomials defining the subarrangements. More precisely, we define the reflection arrangement (after identifying $\left(V^{*}\right)^{*}$ with $V$ ) by the polynomial

$$
Q=Q_{1} \cdots Q_{k} \in S(V) \text {, }
$$

where each $Q_{i} \in S\left(V_{i}\right)$ is the Vandermonde determinant $\left(Q_{i}=\prod_{j_{1}<j_{2}} x_{j_{1}}^{(i)}-x_{j_{2}}^{(i)}\right)$ for $\mathfrak{S}_{n_{i}}$ acting on $V_{i}^{*}$ (see Section 4). As the $\theta_{j}^{(i)}$ are basic derivations, the coefficient matrix of $\left\{\theta_{1}^{(i)}, \ldots, \theta_{n_{i}}^{(i)}\right\}$ has determinant $Q_{i}$ up to a nonzero scalar (by Theorem 4.3). Thus $\theta_{1}^{(1)} \wedge \cdots \wedge \theta_{n_{k}}^{(k)}$ is a nonzero scalar multiple of the form $\left(Q_{1} \cdots Q_{k}\right) x_{1} \wedge \cdots \wedge x_{n}=Q x_{1} \wedge \cdots \wedge x_{n}$. By Proposition 4.3, the derivations $\theta_{j}^{(i)}$ 
wedged together two at a time then generate the invariant derivation 2 -forms over the ring of invariant polynomials.

Contribution from $g$ with $\operatorname{codim} V^{g}=2$.

We now determine the contribution to the Hochschild 2-cohomology of the skew group algebra $S(V) \# G(r, 1, n)$ (under the nonfaithful representation $\rho$ defined in (7.1)) from $g$ with $\operatorname{codim} V^{g}=2$.

Remark 7.5. Suppose $g \in G=G(r, 1, n)$ with $\operatorname{codim}\left(V^{g}\right)=2$ under the nonfaithful action of $G$ on $V$ given by $\rho$. Then for $n \geq 3, g$ is conjugate to a diagonal matrix times either a 3 -cycle or the product of two 2 -cycles (in case $n \geq 4$ ). In fact, we may assume $g$ is conjugate to a diagonal matrix times the permutation

$$
(1,2,3) \quad \text { or } \quad(1,2)(3,4) \text {. }
$$

If $g$ is the product of a diagonal matrix with $(1,2)(3,4)$, then the set of Hochschild semi-invariants for $g$ is zero, i.e. $\operatorname{HH}^{2}(g)=0$ : Suppose $g=\xi_{1}^{a_{1}} \cdots \xi_{n}^{a_{n}}(1,2)(3,4)$ and define $h=\xi_{1}^{a_{1}} \xi_{2}^{a_{2}}(1,2) \in Z(g)$. Then $\operatorname{det}\left(h^{\perp}\right)=-1 \neq 1$ (under the nonfaithful action given by $\rho$ ) and hence $\mathrm{HH}^{2}(g)=0$ by Lemma 3.6 .

In the following proposition, we give explicit invariants by decomposing $V$ into subspaces. Let $V_{A}=\mathbb{C}$-span $\left\{v_{1}, v_{2}, v_{3}\right\}$ and $V_{B}=\mathbb{C}$-span $\left\{v_{4}, \ldots, v_{n}\right\}$. If $g^{\prime}$ is the product of a diagonal matrix and a 3-cycle, then $g^{\prime}$ is conjugate to $g=g_{A} \oplus g_{B}$ where $g_{A} \in G(r, 1,3)$ is a $3 \times 3$ diagonal matrix times the 3 -cycle $(1,2,3)$ and $g_{B}=\xi^{a_{1}} I_{n_{1}} \oplus \cdots \oplus \xi^{a_{k}} I_{n_{k}} \in G(r, 1, n-3)$. (Note that $n_{1}, \ldots, n_{k}$ are simply the multiplicities of the diagonal entries of $g_{B}$.) We apply Lemma 7.2 to $g_{B}$ acting on $V_{B}$ : Rename the basis vectors $v_{4}, \ldots, v_{n}$ of $V_{B}$ so that $v_{1}^{(1)}, \ldots, v_{n_{1}}^{(1)}$ are the first $n_{1}$ basis vectors, $v_{1}^{(2)}, \ldots, v_{n_{2}}^{(2)}$ are the next $n_{2}$ basis vectors, etc.; decompose $V_{B}$ as $V_{1} \oplus \cdots \oplus V_{k}$ with $V_{i}=\mathbb{C}-\operatorname{span}\left\{v_{1}^{(i)}, \ldots, v_{n_{i}}^{(i)}\right\}$.

Proposition 7.6. Assume $n \geq 3$. Let $G=G(r, 1, n)$ act on $V \cong \mathbb{C}^{n}$ via the nonfaithful representation $\rho$, and let $g^{\prime}$ be the product of a diagonal matrix with a 3-cycle. Let $f_{l}^{(i)}$ be the l-th elementary symmetric function in the elements $v_{j}^{(i)}$ defined above. Let $f_{0}=v_{1}+v_{2}+v_{3}$. Then $g^{\prime}$ is conjugate to $g$ with

$$
\mathrm{HH}^{2}(g)=S\left(V^{g}\right)^{\chi}=S\left(V^{g}\right)^{Z(g)}=\mathbb{C}\left[f_{0}, f_{1}^{(1)}, \ldots, f_{n_{1}}^{(1)}, \ldots, f_{1}^{(k)}, \ldots, f_{n_{k}}^{(k)}\right] .
$$

Proof. As in the above paragraph, let $g=g_{A} \oplus g_{B}$. The action of $Z(g)$ on $V=$ $V_{A} \oplus V_{B}$ (and on $\left.V^{g}=V_{A}^{g} \oplus V_{B}^{g}\right)$ decomposes as a direct sum:

$$
Z(g)=Z_{A} \oplus Z_{B}
$$

where $Z_{A}=Z_{G(r, 1,3)}\left(g_{A}\right)$ and $Z_{B}=Z_{G(r, 1, n-3)}\left(g_{B}\right)$. In fact, $Z_{A}=\left\langle\xi I_{3},(1,2,3)\right\rangle$. Under the representation $\rho, g$ acts on $V$ as the permutation $(1,2,3)$. Hence $V^{g}=\mathbb{C}-\operatorname{span}\left\{v_{1}+v_{2}+v_{3}, v_{4}, \ldots, v_{n}\right\}$ and $\left(V^{g}\right)^{\perp}=\mathbb{C}-\operatorname{span}\left\{v_{1}-v_{2}, v_{1}-v_{3}\right\}$. The subgroup $Z(g)$ acts on $\left(V^{g}\right)^{\perp}$ as the group $\langle(1,2,3)\rangle$ with determinant 1 under the representation $\rho$. Thus, the Hochschild character $\chi: Z(g) \rightarrow \mathbb{C}$ (given by $h \mapsto \operatorname{det} h^{\perp}$ ) is trivial (the diagonal matrices act trivially):

$$
S\left(V^{g}\right)^{\chi}=S\left(V^{g}\right)^{Z(g)}
$$

Note that $S\left(V_{A}^{g}\right)=\mathbb{C}\left[v_{1}+v_{2}+v_{3}\right]$ and $Z_{A}$ acts trivially on $v_{1}+v_{2}+v_{3}$. Hence,

$$
S\left(V_{A}^{g}\right)^{Z_{A}}=\mathbb{C}\left[v_{1}+v_{2}+v_{3}\right] .
$$


We apply Lemma 7.2 and Remark 7.4 to the diagonal matrix $g_{B} \in G(r, 1, n-3)$ acting on $V_{B}$ :

$$
S\left(V_{B}^{g}\right)^{Z_{B}}=\mathbb{C}\left[f_{1}^{(1)}, \ldots, f_{n_{1}}^{(1)}, \ldots, f_{1}^{(k)}, \ldots, f_{n_{k}}^{(k)}\right] .
$$

Hence, by Remark 4.1 .

$$
S\left(V^{g}\right)^{Z(g)} \cong S\left(V_{A}^{g}\right)^{Z_{A}} \otimes S\left(V_{B}^{g}\right)^{Z_{B}} \cong \mathbb{C}\left[f_{0}, f_{1}^{(1)}, \ldots, f_{n_{1}}^{(1)}, \ldots, f_{1}^{(k)}, \ldots, f_{n_{k}}^{(k)}\right] .
$$

\section{Putting it together, nonfaithful case.}

We now record the Hochschild 2-cohomology of $S(V) \# G$ where $G=G(r, 1, n)$ acts on $V$ via the nonfaithful representation $\rho$. We combine the contribution to cohomology from $g \in G$ with codim $V^{g}=0$ and the contribution from $g \in G$ with $\operatorname{codim} V^{g}=2$.

Theorem 7.7. Assume $n \geq 3$. Let $G=G(r, 1, n)$ act on $V=\mathbb{C}^{n}$ via the nonfaithful representation $\rho$. The Hochschild cohomology in degree 2 for the skew group algebra $S(V) \# G$ is

$$
\mathrm{HH}^{2}(S(V) \# G) \cong \bigoplus_{g \in C} \mathrm{HH}^{2}(g) \oplus \bigoplus_{g^{\prime} \in C^{\prime}} \mathrm{HH}^{2}\left(g^{\prime}\right)
$$

where

- $C$ is a set of representatives of those conjugacy classes in $G$ containing diagonal matrices, and $\mathrm{HH}^{2}(g)$ is given in Proposition 7.3,

- $C^{\prime}$ is a set of representatives of those conjugacy classes in $G$ containing the product of a diagonal matrix in $G$ with a 3 -cycle, and $\mathrm{HH}^{2}\left(g^{\prime}\right)$ is given in Proposition 7.6 .

Proof. By Equation (3.7),

$$
\mathrm{HH}^{2}(S(V) \# G)=\bigoplus_{\substack{g \in \mathcal{C} \\ \operatorname{codim} V^{g}=0}} \mathrm{HH}^{2}(g) \oplus \bigoplus_{\substack{g^{\prime} \in \mathcal{C} \\ \operatorname{codim} V^{g}=2}} \mathrm{HH}^{2}\left(g^{\prime}\right) .
$$

In Proposition 7.3 we determined the contribution to Hochschild cohomology from those $g$ in $G$ with codim $V^{g}=0$, i.e., from the diagonal matrices in $G$. In Proposition 7.6. we determined the contribution to Hochschild cohomology from those $g$ in $G$ with $\operatorname{codim} V^{g}=2$, i.e., from those $g \in G$ conjugate to the product of a diagonal matrix in $G$ and a 3 -cycle.

\section{Graded Hecke algebras as deformations of $S(V) \# G$}

In this section, we define a graded Hecke algebra associated to a finite group $G$ and any finite dimensional representation $V$ of $G$. We give an explicit connection between graded Hecke algebras and the Hochschild semi-invariants defined in Section 3. As a consequence of Theorems 5.1] and 8.7, if $G=G(r, p, n)$ with $r \geq 3, n \geq 4$, and $V$ is its natural reflection representation, then there are no nontrivial graded Hecke algebras: The relevant Hochschild cohomology, while nonzero, is not of the required form. This nonexistence of a graded Hecke algebra was discovered by Ram and the first author [22], and now we may view their result in the context of algebraic deformation theory and Hochschild cohomology. This negative result inspired their ad hoc construction of a "different graded Hecke algebra" for $G(r, 1, n)$, which coincides with an algebra defined by Dezélée [7] in case $r=2$. 
In the next section, we show that this "different graded Hecke algebra" is in fact a graded Hecke algebra under our broader Definition 8.1 in which we allow nonfaithful actions of $G$. This also motivates our earlier computations in Section 7 of Hochschild cohomology for a nonfaithful action of $G(r, 1, n)$ on $V=\mathbb{C}^{n}$ : Theorems 7.7 and 8.7 imply existence of a generic graded Hecke algebra depending on many parameters, described in (9.1).

The following definition includes the symplectic reflection algebras defined by Chmutova [6] in case $G$ acts symplectically, i.e., when there is a (not necessarily injective) group homomorphism $G \rightarrow \operatorname{Sp}(V)$. For brevity, we omit tensor symbols in expressions in the tensor algebra $T(V)$.

Definition 8.1. Let $G$ be a finite group with a representation $V=\mathbb{C}^{n}$. For each $g \in G$, choose a skew-symmetric bilinear form $a_{g}: V \times V \rightarrow \mathbb{C}$. Let

$$
A=(T(V) \# G) /\left(v w-w v-\sum_{g \in G} a_{g}(v, w) \bar{g}\right),
$$

where we quotient by the ideal generated by all elements of the given form for $v, w \in$ $V$. Consider $A$ to be a filtered algebra in which we assign degree 1 to elements of $V$ and degree 0 to elements of $G$. We call $A$ a graded Hecke algebra if the associated graded algebra gr $A$ is isomorphic to $S(V) \# G$. Equivalently $A$ is isomorphic as a vector space to $S(V) \# G$ via a choice of linear section $S(V) \hookrightarrow T(V)$ of the canonical projection of $T(V)$ onto $S(V)$.

In case $G$ is a Coxeter group and $V$ its natural reflection representation, the graded algebra associated to the affine Hecke algebra (with respect to a different choice of filtration) defined by Lusztig [18] is a graded Hecke algebra under our definition [22, §3]. This is the origin of the term graded Hecke algebra.

By definition, graded Hecke algebras are parametrized by sets $\left\{a_{g}\right\}_{g \in G}$ satisfying suitable conditions. These conditions may be determined by applying [22, Lemma 1.5], valid in this more general setting when the action of $G$ on $V$ may not be faithful:

Lemma 8.2. Let $A$ be the algebra defined by a set of skew-symmetric bilinear forms $\left\{a_{g}\right\}_{g \in G}$ in Definition 8.1. Then $A$ is a graded Hecke algebra if and only if

$$
\begin{gathered}
\bar{h}[v, w](\bar{h})^{-1}=[h(v), h(w)], \quad \text { and } \\
{[u,[v, w]]+[v,[w, u]]+[w,[u, v]]=0}
\end{gathered}
$$

in $A$, for all $u, v, w \in V$ and $h \in G$ (where $[v, w]=v w-w v)$.

Equations (8.3) and (8.4) are equivalent to the uniqueness of the expressions $\bar{h} w v$ and $w v u$ when rearranged with all group elements to the right and vector space elements in alphabetical order. In case $G$ acts faithfully on $V$, this lemma was used in 22 to show directly that the sets $\left\{a_{g}\right\}_{g \in G}$ corresponding to graded Hecke algebras form a vector space of dimension $d+\operatorname{dim}\left(\bigwedge^{2}(V)\right)^{G}$, where $d$ is the number of conjugacy classes of $g \in G$ such that $\operatorname{codim}\left(V^{g}\right)=2$ and $\chi_{g} \equiv 1$ (where $\chi_{g}(h)=\operatorname{det}\left(h^{\perp}\right)$ for $\left.h \in Z(g)\right)$. This approach may be adapted to nonfaithful actions to yield Corollary 8.17 below. However, we take a somewhat different route in the next theorem in order to relate graded Hecke algebras to Hochschild cohomology, and thus to other potential deformations of $S(V) \# G$. 
Definition 8.5. Let $g \in G$ and $f_{g} \in \mathrm{HH}^{2}(g)$, i.e., $f_{g}$ is a Hochschild semi-invariant of $g\left(\right.$ see (3.2)). By definition, we may write $f_{g}$ as a linear combination of elements of the form $p \otimes y$, where $p \in S\left(V^{g}\right), y \in \Lambda\left(\left(V^{g}\right)^{*}\right)$. We say that $f_{g}$ has degree $m$ if the polynomial $p$ of highest degree in such a linear combination has degree $m$ (with respect to fixed bases of $\left.V^{g}, \bigwedge\left(\left(V^{g}\right)^{*}\right)\right)$. Note that the homogeneous parts of $f_{g}$ are also Hochschild semi-invariants of $g$.

Remark 8.6. In the next theorem, we explain how to define a graded Hecke algebra from an element of Hochschild cohomology. We first outline how one identifies a Hochschild semi-invariant with a function on $S(V)^{e} \otimes \bigwedge^{2}(V)$ (recall $S(V)^{e}=$ $\left.S(V) \otimes S(V)^{o p}\right)$. Let $\mathcal{C}$ denote a set of representatives of the conjugacy classes of $G$, as before. Via (3.7), each element of $\operatorname{HH}^{2}(S(V) \# G)$ may be identified with a set $\left\{f_{g}\right\}_{g \in \mathcal{C}}$ of Hochschild semi-invariants (see (3.2)). Applying (3.4) as well, we regard each $f_{g}$ as an element of $S\left(V^{g}\right) \bar{g} \otimes \Lambda^{2}\left(V^{*}\right)$ by making the canonical identification of the vector space $\bigwedge^{2-\operatorname{codim} V^{g}}\left(\left(V^{g}\right)^{*}\right) \otimes \bigwedge^{\operatorname{codim} V^{g}}\left(\left(\left(V^{g}\right)^{\perp}\right)^{*}\right)$ with a subspace of $\Lambda^{2}\left(V^{*}\right)$ and by inserting the factor $\bar{g}$. This allows us to consider $f_{g}$ as a $Z(g)$-invariant function on $S(V)^{e} \otimes \bigwedge^{2}(V)$ in the following way: Suppose $f_{g}$ is a linear combination of elements of the form $p \bar{g} \otimes y\left(p \in S\left(V^{g}\right), y \in \bigwedge^{2}\left(V^{*}\right)\right)$. Let $r, s \in S(V), z \in \bigwedge^{2}(V)$. Then $f_{g}(r \otimes s \otimes z) \in S(V) \bar{g}$ is the corresponding linear combination of elements $y(z) r p \bar{g} s$ (see the proof of Theorem 8.7 below for details). We further apply a transfer (trace) operator to each $f_{g}$ to obtain the corresponding $G$-invariant function $f$ on $S(V)^{e} \otimes \bigwedge^{2}(V)$ :

$$
f=\sum_{g \in \mathcal{C}} \sum_{h \in G / Z(g)} h\left(f_{g}\right),
$$

where $\left(h\left(f_{g}\right)\right)(r \otimes s \otimes z)=h\left(f_{g}\left(h^{-1}(r \otimes s \otimes z)\right)\right)$.

Theorem 8.7. Let $G$ be a finite group with a representation $V=\mathbb{C}^{n}$. The parameter space of graded Hecke algebras for the pair $G, V$ is isomorphic to the space consisting of sets $\left\{f_{g}\right\}_{g \in G}$ of Hochschild semi-invariants whose nonzero elements have degree 0. The defining skew-symmetric bilinear forms $\left\{a_{g}\right\}_{g \in G}$ of the graded Hecke algebra corresponding to $\left\{f_{g}\right\}_{g \in \mathcal{C}}$ are given by $a_{g}(v, w) \bar{g}=f_{g}(1 \otimes 1 \otimes v \wedge w)$ and $a_{h^{-1} g h}(v, w)=a_{g}(h(v), h(w))$ for all $g \in \mathcal{C}, h \in G$, and $v, w \in V$.

The remainder of this section is devoted to proving this theorem and its corollary. We need some technical lemmas and formulas from [5, 26, 27. We obtain the forms $a_{g}$ from the functions $f_{g}$ by finding intermediary Hochschild two-cocycles $\mu_{1}$ and corresponding deformations of $S(V) \# G$. First, we recall the definitions of deformations and Hochschild two-cocycles. For more details, see [13] or [10].

Let $t$ be an indeterminate. If $R$ is any associative $\mathbb{C}$-algebra (such as $R=$ $S(V) \# G)$, a deformation of $R$ over $\mathbb{C}[t]$ consists of the $\mathbb{C}[t]$-module $R[t]=$ $\mathbb{C}[t] \otimes R$ together with an associative product $*$ of the form

$$
a * b=a b+\mu_{1}(a, b) t+\mu_{2}(a, b) t^{2}+\cdots
$$

for all $a, b \in R$, where $a b$ is the product of $a$ and $b$ in $R$ and $\mu_{i}: R \times R \rightarrow R$ is $\mathbb{C}$-bilinear (extended to be $\mathbb{C}[t]$-bilinear) for each $i$. (In order for $a * b$ to be in $R[t]$, this sum must in fact be finite for each pair $a, b$; one may also be interested in deformations over $\mathbb{C}[[t]]$ or another extension of $\mathbb{C}$, but we will not need these here.) 
Associativity implies conditions on the $\mu_{i}$. In particular $\mu_{1}$ must be a Hochschild two-cocycle; i.e.,

$$
\mu_{1}(a, b c)+a \mu_{1}(b, c)=\mu_{1}(a b, c)+\mu_{1}(a, b) c
$$

for all $a, b, c \in R$. That is, $\mu_{1}$ is a representative of an element of $\mathrm{HH}^{2}(R)$ obtained via the bar complex

$$
\cdots \stackrel{\delta_{3}}{\longrightarrow} R^{\otimes 4} \stackrel{\delta_{2}}{\longrightarrow} R^{\otimes 3} \stackrel{\delta_{1}}{\longrightarrow} R^{e} \stackrel{m}{\longrightarrow} R \rightarrow 0,
$$

where $\delta_{i}\left(a_{0} \otimes a_{1} \otimes \cdots \otimes a_{i+1}\right)=\sum_{j=0}^{i}(-1)^{j} a_{0} \otimes \cdots \otimes a_{j} a_{j+1} \otimes \cdots \otimes a_{i+1}$ and $m$ is multiplication. This is an $R^{e}$-free resolution of $R$ (where $R^{e}=R \otimes R^{o p}$ ), and thus yields $\mathrm{HH}^{*}(R)=\operatorname{Ext}_{R^{e}}(R, R)$ upon taking cohomology of the cochain complex resulting from application of $\operatorname{Hom}_{R^{e}}(R,-)$. Specifically, $\operatorname{HH}^{i}(R)=\operatorname{Ker}\left(\delta_{i+1}^{*}\right) / \operatorname{Im}\left(\delta_{i}^{*}\right)$, defined via the cochain complex

$$
0 \rightarrow \operatorname{Hom}_{R^{e}}\left(R^{e}, R\right) \stackrel{\delta_{1}^{*}}{\longrightarrow} \operatorname{Hom}_{R^{e}}\left(R^{\otimes 3}, R\right) \stackrel{\delta_{2}^{*}}{\longrightarrow} \operatorname{Hom}_{R^{e}}\left(R^{\otimes 4}, R\right) \stackrel{\delta_{3}^{*}}{\longrightarrow} \cdots .
$$

We identify $\operatorname{Hom}_{R^{e}}\left(R^{\otimes 4}, R\right) \cong \operatorname{Hom}_{\mathbb{C}}\left(R^{\otimes 2}, R\right)$, and a straightforward calculation yields Equation (8.9) as the defining relation for elements of $\operatorname{Ker}\left(\delta_{3}^{*}\right)$.

In order to prove Theorem 8.7 we explain how to obtain the defining skewsymmetric forms $\left\{a_{g}\right\}_{g \in G}$ of a graded Hecke algebra from a set of Hochschild semiinvariants $\left\{f_{g}\right\}_{g \in \mathcal{C}}$ whose nonzero elements have degree 0 . The following comparison of the bar complex (8.10) to a Koszul complex allows us to write down corresponding Hochschild 2-cocycles $\mu_{1}$ explicitly.

There is a chain map from the bar complex (8.10) for $R=S(V)$ to the Koszul complex $P_{\bullet}=K\left(\left\{v_{i} \otimes 1-1 \otimes v_{i}\right\}_{i=1}^{n}\right)$, where $v_{1}, \ldots, v_{n}$ is a basis of $V$ (see [25, §4.5] for details on Koszul complexes):

$$
\begin{array}{ccccccccccc}
\cdots & \rightarrow & S(V)^{\otimes 4} & \stackrel{\delta_{2}}{\longrightarrow} & S(V)^{\otimes 3} & \stackrel{\delta_{1}}{\longrightarrow} & S(V)^{e} & \stackrel{m}{\longrightarrow} & S(V) & \rightarrow & 0 \\
& & \downarrow \psi_{2} & & \downarrow \psi_{1} & & \| & & \| & & \\
\cdots & \rightarrow & S(V)^{e} \otimes \bigwedge^{2}(V) & \stackrel{d_{2}}{\longrightarrow} & S(V)^{e} \otimes \Lambda^{1}(V) & \stackrel{d_{1}}{\longrightarrow} & S(V)^{e} & \stackrel{m}{\longrightarrow} & S(V) & \rightarrow & 0
\end{array}
$$

The differentials $d_{1}, d_{2}$ are $S(V)^{e}$-homomorphisms given in our notation by

$$
\begin{aligned}
d_{1}\left(1 \otimes 1 \otimes v_{i}\right) & =v_{i} \otimes 1-1 \otimes v_{i}, \\
d_{2}\left(1 \otimes 1 \otimes v_{i} \wedge v_{j}\right) & =\left(v_{i} \otimes 1-1 \otimes v_{i}\right) \otimes v_{j}-\left(v_{j} \otimes 1-1 \otimes v_{j}\right) \otimes v_{i},
\end{aligned}
$$

for $1 \leq i<j \leq n$. The vertical maps $\psi_{1}$ and $\psi_{2}$ may be given by the formulas (see [26. (4.9) and (4.10)], where the notation is somewhat different):

$$
\psi_{1}\left(1 \otimes v_{1}^{k_{1}} \cdots v_{n}^{k_{n}} \otimes 1\right)=\sum_{i=1}^{n} \sum_{a=1}^{k_{i}} v_{i}^{k_{i}-a} v_{i+1}^{k_{i+1}} \cdots v_{n}^{k_{n}} \otimes v_{1}^{k_{1}} \cdots v_{i-1}^{k_{i-1}} v_{i}^{a-1} \otimes v_{i},
$$

$$
\begin{aligned}
& \psi_{2}\left(1 \otimes v_{1}^{k_{1}} \cdots v_{n}^{k_{n}} \otimes v_{1}^{m_{1}} \cdots v_{n}^{m_{n}} \otimes 1\right) \\
&=\sum_{1 \leq i<j \leq n} \sum_{b=1}^{m_{j}} \sum_{a=1}^{k_{i}} v_{i}^{k_{i}-a} v_{i+1}^{k_{i+1}} \cdots v_{j-1}^{k_{j-1}} v_{j}^{k_{j}+m_{j}-b} v_{j+1}^{k_{j+1}+m_{j+1}} \cdots v_{n}^{k_{n}+m_{n}} \\
& \quad \otimes v_{1}^{k_{1}+m_{1}} \cdots v_{i-1}^{k_{i-1}+m_{i-1}} v_{i}^{m_{i}+a-1} v_{i+1}^{m_{i+1}} \cdots v_{j-1}^{m_{j-1}} v_{j}^{b-1} \otimes v_{i} \wedge v_{j} .
\end{aligned}
$$

To obtain an explicit Hochschild two-cocycle $\mu_{1}$ from a set $\left\{f_{g}\right\}_{g \in \mathcal{C}}$ of Hochschild semi-invariants, we apply the following proposition. This proposition appears as 
part of [5, Thm. 5.4], where it is stated for arbitrary degree and arbitrary resolution $P$. It is valid also when $G$ acts nonfaithfully on $V$.

Proposition 8.13. Let $R=S(V) \# G$. Let $f: S(V)^{e} \otimes \bigwedge^{2}(V) \rightarrow R$ be a function (on the degree 2 term of the above Koszul complex) representing an element of $\mathrm{HH}^{2}(S(V), R)^{G}$. Under the isomorphism $\mathrm{HH}^{2}(S(V), R)^{G} \cong \mathrm{HH}^{2}(R), f$ is mapped to the function $\mu_{1} \in \operatorname{Hom}_{\mathbb{C}}\left(R^{\otimes 2}, R\right) \cong \operatorname{Hom}_{R^{e}}\left(R^{\otimes 4}, R\right)$ whose action on the degree 2 term of the bar complex (8.10) is given by

$$
\mu_{1}(r \bar{g} \otimes s \bar{h})=\left(\left(f \circ \psi_{2}\right)(1 \otimes r \otimes g(s) \otimes 1)\right) \overline{g h},
$$

for all $r, s \in S(V)$ and $g, h \in G$.

Our final tool for proving Theorem 8.7 is [27, Thm. 3.2], valid when $G$ acts nonfaithfully on $V$; we also record it here for convenience. Consider $S(V) \# G$ to be a graded algebra where elements of $V$ have degree 1 and elements of $G$ have degree 0 . In [27, a graded Hecke algebra is defined over $\mathbb{C}[t]$ : It is a quotient of $T(V) \# G[t]$ by the ideal generated by all $v w-w v-\sum_{g \in G} a_{g}(v, w) t \bar{g}$, whose associated graded $\mathbb{C}[t]-$ algebra is isomorphic to $S(V) \# G[t]$. To obtain our Definition 8.1, simply substitute any nonzero complex number for $t$.

Proposition 8.14. Up to isomorphism, the (nontrivial) graded Hecke algebras over $\mathbb{C}[t]$ are precisely the deformations of $S(V) \# G$ over $\mathbb{C}[t]$ for which $\operatorname{deg} \mu_{i}=-2 i$ $(i \geq 1)$.

Proof of Theorem 8.7. Suppose $\left\{f_{g}\right\}_{g \in \mathcal{C}}$ is a set of Hochschild semi-invariants and that each nonzero $f_{g}$ has degree 0. As in Remark 8.6, let $f$ be the associated function on $S(V)^{e} \otimes \bigwedge^{2}(V)$. Let $\mu_{1}$ be the corresponding Hochschild 2-cocycle of $S(V) \# G$, given by Proposition 8.13. Let $v, w \in V$. By Remark 8.6. Proposition 8.13, and formula (8.12), $\mu_{1}(v \otimes w)=\left(f \circ \psi_{2}\right)(1 \otimes v \otimes w \otimes 1)$ is an element of the subalgebra $\mathbb{C} G$ of $S(V) \# G$. Similarly, $\mu_{1}(w \otimes v)$ is an element of $\mathbb{C} G$. Define scalars $a_{g}(v, w)$ by setting

$$
\mu_{1}(v \otimes w)-\mu_{1}(w \otimes v)=\sum_{g \in G} a_{g}(v, w) \bar{g} .
$$

As $\mu_{1}$ is bilinear, each resulting function $a_{g}: V \times V \rightarrow \mathbb{C}$ is bilinear. By definition, $a_{g}$ is skew-symmetric for each $g \in G$. We claim that the set $\left\{a_{g}\right\}_{g \in G}$ defines a graded Hecke algebra. Let $A$ be the corresponding quotient given in Definition 8.1. By Lemma 8.2, it suffices to verify (8.3) and (8.4) for $A$.

By Definition 8.1 and Equation (8.15), $[v, w]=\mu_{1}(v \otimes w)-\mu_{1}(w \otimes v)$ lies in $A$, for all $v, w \in V$. Abuse notation and write $\psi_{2}(v \otimes w)=\psi_{2}(1 \otimes v \otimes w \otimes 1)$. Apply definition (8.12) of $\psi_{2}$ to our chosen basis for $V$ to see that $\psi_{2}\left(v_{i} \otimes v_{j}\right)=1 \otimes 1 \otimes v_{i} \wedge v_{j}$ if $i<j$ and 0 otherwise. After expressing all vectors in terms of this basis, we find

$$
h\left(\psi_{2}(v \otimes w)-\psi_{2}(w \otimes v)\right)=\psi_{2}(h(v) \otimes h(w))-\psi_{2}(h(w) \otimes h(v))
$$

for all $v, w \in V, h \in G$. As $f$ is also $G$-invariant, it follows that

$$
\mu_{1}(v \otimes w)-\mu_{1}(w \otimes v)=\left(f \circ \psi_{2}\right)(v \otimes w)-\left(f \circ \psi_{2}\right)(w \otimes v)
$$

is $G$-invariant as a function on $v, w \in V$, and thus (8.3) holds in $A$.

Next we claim that the Jacobi identity (8.4) is a direct consequence of the Hochschild 2-cocycle condition (8.9). The left side of (8.4) may be rewritten by 
replacing the innermost bracket $[v, w]$ in the first term by $\mu_{1}(v \otimes w)-\mu_{1}(w \otimes v)$, and similarly for each of the other two terms. We obtain

$$
\begin{aligned}
& u \mu_{1}(v \otimes w)-u \mu_{1}(w \otimes v)-\mu_{1}(v \otimes w) u \\
& \quad+\mu_{1}(w \otimes v) u+v \mu_{1}(w \otimes u)-v \mu_{1}(u \otimes w)-\mu_{1}(w \otimes u) v+\mu_{1}(u \otimes w) v \\
& \quad+w \mu_{1}(u \otimes v)-w \mu_{1}(v \otimes u)-\mu_{1}(u \otimes v) w+\mu_{1}(v \otimes u) w .
\end{aligned}
$$

Substitutions from the six permutations of (8.9) in which $\{a, b, c\}=\{u, v, w\}$ yield

$$
\begin{aligned}
& \mu_{1}(u v \otimes w)-\mu_{1}(u \otimes v w)+\mu_{1}(u \otimes w v)-\mu_{1}(u w \otimes v) \\
& \quad+\mu_{1}(v w \otimes u)-\mu_{1}(v \otimes w u)+\mu_{1}(w \otimes v u)-\mu_{1}(w v \otimes u) \\
& \quad+\mu_{1}(v \otimes u w)-\mu_{1}(v u \otimes w)+\mu_{1}(w u \otimes v)-\mu_{1}(w \otimes u v) .
\end{aligned}
$$

But this expression is zero as $S(V)$ is commutative. We have thus shown that (8.4) holds in $A$. This concludes the proof that a set of Hochschild semi-invariants whose nonzero elements have degree 0 gives rise to a graded Hecke algebra.

We now argue that every graded Hecke algebra arises in this way. Note that if a set $\left\{a_{g}\right\}_{g \in G}$ of skew-symmetric bilinear forms defines a graded Hecke algebra, then so does $\left\{\alpha a_{g}\right\}_{g \in G}$ for any fixed scalar $\alpha$. We may therefore consider the related graded Hecke algebra over $\mathbb{C}[t]$, where the indeterminate $t$ takes the place of the arbitrary scalar $\alpha$. By Proposition 8.14, a (nontrivial) graded Hecke algebra over $\mathbb{C}[t]$ is a deformation of $S(V) \# G$ such that the associated Hochschild 2-cocycle $\mu_{1}$ satisfies $\operatorname{deg} \mu_{1}=-2$, and more generally $\operatorname{deg}\left(\mu_{i}\right)=-2 i(i \geq 1)$. Equation (8.8) and Definition 8.1 (with $a_{g}$ replaced by $t a_{g}$ ) force relationship (8.15) between the defining forms $t a_{g}$ and the cocycle $\mu_{1}$. As $\mu_{1}$ is a Hochschild 2-cocycle, Equation (3.7) implies the existence of a set of Hochschild semi-invariants $\left\{f_{g}\right\}_{g \in \mathcal{C}}$ and a corresponding function $f$ (see Remark 8.6) such that $\psi_{2}^{*}(f)$ is cohomologous to $\mu_{1}$. In particular, there exists an $S(V)^{e}$-homomorphism $\beta: S(V)^{\otimes 3} \rightarrow S(V) \# G$ such that $f \circ \psi_{2}=\mu_{1}+\beta \circ \delta_{2}$. Identify $\beta$ with a $\mathbb{C}$-linear function from $S(V)$ to $S(V) \# G$, and note that

$$
\beta\left(\delta_{2}(1 \otimes v \otimes w \otimes 1)\right)=v \beta(w)-\beta(v w)+\beta(v) w
$$

for all $v, w \in V$. Thus for all $i<j$, we have

$\left(f \circ \psi_{2}\right)\left(v_{i} \otimes v_{j}\right)=\mu_{1}\left(v_{i} \otimes v_{j}\right)-\mu_{1}\left(v_{j} \otimes v_{i}\right)+v_{i} \beta\left(v_{j}\right)+\beta\left(v_{i}\right) v_{j}-v_{j} \beta\left(v_{i}\right)-\beta\left(v_{j}\right) v_{i}$

(since $\psi_{2}\left(v_{j} \otimes v_{i}\right)=0$ when $\left.i<j\right)$. Thus $\left(f \circ \psi_{2}\right)\left(v_{i} \otimes v_{j}\right)$ is a sum of the element $\mu_{1}\left(v_{i} \otimes v_{j}\right)-\mu_{1}\left(v_{j} \otimes v_{i}\right)$ in $\mathbb{C} G$ and the element $v_{i} \beta\left(v_{j}\right)+\beta\left(v_{i}\right) v_{j}-v_{j} \beta\left(v_{i}\right)-\beta\left(v_{j}\right) v_{i}$ in the ideal $(V)$ of $S(V) \# G$. Now $\left(f \circ \psi_{2}\right)\left(v_{i} \otimes v_{j}\right)=f\left(1 \otimes 1 \otimes v_{i} \wedge v_{j}\right)$, and the function $f$ is discussed in Remark 8.6, in particular it is determined by these values since it is an $S(V)^{e}$-homomorphism. Since $S(V) \# G \cong \mathbb{C} G \oplus S(V)$ as a vector space and the homogeneous parts of $f$ are also $G$-invariant functions on $S(V)^{e} \otimes \bigwedge^{2}(V)$, we have $f=f^{\prime}+f^{\prime \prime}$ where $\left(f^{\prime} \circ \psi_{2}\right)\left(v_{i} \otimes v_{j}\right)=\mu_{1}\left(v_{i} \otimes v_{j}\right)-\mu_{1}\left(v_{j} \otimes v_{i}\right)$ and $f^{\prime \prime} \circ \psi_{2}=\beta \circ \delta_{2}$ is a coboundary. By Remark 8.6. $f^{\prime}$ itself corresponds to a set of Hochschild semi-invariants, $\left\{f_{g}^{\prime}\right\}_{g \in \mathcal{C}}$. As $\operatorname{deg} \mu_{1}=-2$ and $\operatorname{deg} \psi_{2}=-2$, each $f_{g}^{\prime}$ is either 0 or has degree 0 . Thus $\left\{f_{g}^{\prime}\right\}_{g \in \mathcal{C}}$ is the desired set of Hochschild semiinvariants. (Alternatively, we could simply prove that our map from sets $\left\{f_{g}\right\}_{g \in \mathcal{C}}$ of Hochschild semi-invariants (whose nonzero elements have degree 0) to sets $\left\{a_{g}\right\}_{g \in G}$ of skew-symmetric forms defining graded Hecke algebras is surjective by classifying the latter directly from Lemma 8.2, cf. [22, Thm. 1.9].) 
The formula for the $a_{g}$ in terms of the $f_{g}$ follows from our calculations above: If $i<j$, then

$$
\begin{aligned}
\sum_{g \in G} a_{g}\left(v_{i}, v_{j}\right) \bar{g}=\left[v_{i}, v_{j}\right] & =\mu_{1}\left(v_{i} \otimes v_{j}\right)-\mu_{1}\left(v_{j} \otimes v_{i}\right) \\
& =\left(f \circ \psi_{2}\right)\left(v_{i} \otimes v_{j}\right)=f\left(1 \otimes 1 \otimes v_{i} \wedge v_{j}\right) .
\end{aligned}
$$

Now examine the coefficient of each $\bar{g}(g \in G)$ separately, and apply (8.3). The resulting formula extends from pairs $v_{i}, v_{j}$ of basis elements to all $v, w \in V$ by linearity.

Remark 8.16. We point out an interesting consequence of the Hochschild 2-cocycle condition (8.9) in this context: Let $\mu_{1}$ and $A$ be as in the first paragraph of the proof of Theorem 8.7. We showed that the Jacobi identity (8.4) holds in $A$ as a direct consequence of the Hochschild 2-cocycle condition (8.9) for $\mu_{1}$.

The following corollary characterizes graded Hecke algebras without reference to Hochschild cohomology. Recall that $\chi_{g}(h)=\operatorname{det}\left(h^{\perp}\right)$ for $h \in Z(g)$, where $h^{\perp}=\left.h\right|_{\left(V^{g}\right) \perp}$, and $\mathcal{C}$ denotes a set of representatives of the conjugacy classes of $G$.

Corollary 8.17. Let $d$ be the number of conjugacy classes of $g \in G$ such that $\operatorname{codim} V^{g}=2$ and $\chi_{g} \equiv 1$.

(i) The sets $\left\{a_{g}\right\}_{g \in G}$ corresponding to graded Hecke algebras form a vector space of dimension

$$
d+\sum_{\substack{g \in \mathcal{C} \\ V^{g}=V}} \operatorname{dim}\left(\bigwedge^{2}(V)\right)^{Z(g)} .
$$

(ii) All graded Hecke algebras arise in the following way: For each $g \in \mathcal{C}$ satisfying $\operatorname{codim} V^{g}=2$ and $\chi_{g} \equiv 1$, define a skew-symmetric bilinear form $a_{g}: V \times V \rightarrow \mathbb{C}$ by setting $a_{g}\left(w_{1}, w_{2}\right)$ equal to an arbitrary scalar for a fixed basis $\left\{w_{1}, w_{2}\right\}$ of $\left(V^{g}\right)^{\perp}, a_{g}\left(V^{g}, V\right)=0$, and $a_{h^{-1} g h}(v, w)=a_{g}(h(v), h(w))$ for all $h \in G, v, w \in V$. For each $g \in \mathcal{C}$ satisfying $V^{g}=V$, define $a_{g}$ by any choice of $Z(g)$-invariant linear functional on $\bigwedge^{2}(V)$, and $a_{h^{-1} g h}(v, w)=$ $a_{g}(h(v), h(w))$ for all $h \in G, v, w \in V$.

Proof. By (3.7), if $\mathrm{HH}^{2}(g)$ is nonzero, then codim $V^{g} \in\{0,2\}$. We apply Theorem 8.7 in each case. If codim $V^{g}=0$ (i.e. $V^{g}=V$ ), the Hochschild semi-invariants of $g$ that are either 0 or of degree 0 form a vector space of dimension $\operatorname{dim}\left(\bigwedge^{2}(V)\right)^{Z(g)}$ (see (3.2)). Theorem 8.7. Remark 8.6] and (8.3) then give the indicated form of the corresponding functions $a_{g}$. If $\operatorname{codim} V^{g}=2$, a nonzero scalar is a Hochschild semi-invariant for $g$ if and only if $\chi_{g} \equiv 1$ (by definition). Again by Theorem 8.7. Remark 8.6 and (8.3), the values of the corresponding forms $a_{g}$ are precisely those given in (ii). (Alternatively the corollary may be proven directly, without using Hochschild cohomology; cf. [22, Thm. 1.9]).

\section{Graded Hecke algebras for $G(r, 1, n)$}

Assume $n \geq 3$. Let $G=G(r, 1, n)$ act on $V=\mathbb{C}^{n}$ nonfaithfully as the symmetric group via $\rho$ defined in (7.1). In this section, we find all graded Hecke algebras corresponding to this action. Similar results hold for $G(r, p, n)$. We prove that in a special case, these algebras are isomorphic to algebras that appeared in 22, and in [7] when $r=2$. 
By Theorems 7.7 and 8.7, the parameter space of graded Hecke algebras for $G, V$ has dimension equal to the number of conjugacy classes of elements $g$ in $G(r, 1, n)$ for which $g$ is the product of a diagonal matrix with a 3-cycle. (By Proposition 7.3 and Remark 7.4, the diagonal matrices themselves do not have Hochschild semiinvariants of degree 0.) We refine the notation introduced in the text preceding

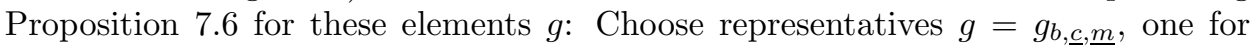
each conjugacy class, where

$$
g_{b, \underline{c}, \underline{m}}=\xi_{3}^{b}(1,2,3) \xi^{c_{1}} I_{m_{1}} \oplus \cdots \oplus \xi^{c_{k}} I_{m_{k}},
$$

$\underline{c}=\left(c_{1}, \ldots, c_{k}\right), \underline{m}=\left(m_{1}, \ldots, m_{k}\right)$, and $0 \leq b, c_{1}, \ldots, c_{k} \leq r-1\left(m_{1}, \ldots, m_{k}\right.$ are the multiplicities of the diagonal entries other than the first three). By Corollary 8.17(ii), all graded Hecke algebras arise as follows. For each representative $g_{b, \underline{c}, \underline{m}}$, choose a scalar $\alpha_{b, \underline{c}, \underline{m}}$. The corresponding graded Hecke algebra is

$$
A=(T(V) \# G) /\left(v w-w v-\sum_{g \in G(r, 1, n)} a_{g}(v, w) \bar{g}\right),
$$

where the skew-symmetric bilinear forms $a_{g}(g \in G)$ are given by:

- $a_{g} \equiv 0$ if $g$ is not conjugate to one of the representatives $g_{b, \underline{c}, \underline{m}}$;

- $a_{g_{b, \underline{c}, \underline{m}}}\left(v_{1}-v_{2}, v_{2}-v_{3}\right)=\alpha_{b, \underline{c}, \underline{m}}$ and $a_{g_{b, \underline{c}, \underline{m}}}\left(V^{(1,2,3)}, V\right)=0$;

- $a_{h^{-1} g_{b, \underline{c}, \underline{\underline{m}}} h}(v, w)=a_{g_{b, \underline{c}, \underline{\underline{m}}}}(h(v), h(w))$ for all $h \in G$.

We are particularly interested in the choice $\alpha_{0,0, n-3}=1(k=1)$ and all other $\alpha_{b, \underline{c}, \underline{m}}=0$. Denote the resulting graded Hecke algebra by $A_{r, 1, n}$ :

Definition 9.2. $A_{r, 1, n}=(T(V) \# G(r, 1, n)) /\left(v w-w v-\sum_{g \in G(r, 1, n)} a_{g}(v, w) \bar{g}\right)$, where the skew-symmetric bilinear forms $a_{g}(g \in G)$ are given by:

- $a_{g} \equiv 0$ if $g$ is not conjugate to $(1,2,3)$;

- $a_{(1,2,3)}\left(v_{1}-v_{2}, v_{2}-v_{3}\right)=1$ and $a_{(1,2,3)}\left(V^{(1,2,3)}, V\right)=0$;

- $a_{h^{-1}(1,2,3) h}(v, w)=a_{(1,2,3)}(h(v), h(w))$ for all $h \in G$.

We claim that the defining relations $v w-w v-\sum_{g \in G} a_{g}(v, w) \bar{g}$ of $A_{r, 1, n}$ may be replaced by

$$
v_{m} v_{k}-v_{k} v_{m}=\frac{1}{3} \sum_{\substack{1 \leq i \leq n \\ i \neq m, k}} \sum_{\substack{a, b=0 \\ r-1}} \bar{\xi}_{m}^{a} \bar{\xi}_{k}^{b}\left(\bar{\xi}_{i}\right)^{-a-b}((\overline{m, k, i})-(\overline{m, i, k}))
$$

for all $1 \leq m<k \leq n$. To see this, first note that the group elements in this sum are precisely those that both are conjugate to $(1,2,3)$ and act nontrivially on $v_{m}, v_{k}$. For each $i, a, b$ let $h$ be an element of $\mathfrak{S}_{n}$ such that $h^{-1}(1,2,3) h=\xi_{m}^{a} \xi_{k}^{b} \xi_{i}^{-a-b}(m, k, i)$ (or $\left.h^{-1}(1,2,3) h=\xi_{m}^{a} \xi_{k}^{b} \xi_{i}^{-a-b}(m, i, k)\right)$. Then apply Definition 9.2, writing $v_{1}, v_{2}, v_{3}$ in terms of the vector space decomposition $V \cong\left(V^{(1,2,3)}\right)^{\perp} \oplus V^{(1,2,3)}$.

We will show that the graded Hecke algebra $A_{r, 1, n}$ is precisely the algebra defined by Ram and the first author [22, (5.1)] as a substitute for a (nonexistent) graded Hecke algebra corresponding to the natural reflection representation of $G(r, 1, n)$. Their algebra agrees with one defined by Dezélée [7 in the case $r=2$. Our results 
in Section 8 will then imply that these algebras of [7, 22] arise from deformations of $S(V) \# G(r, 1, n)$ over $\mathbb{C}[t]$. They were originally given in a different form, analogous to Lusztig's definition of a graded Hecke algebra. The proof that Lusztig's definition is a special case of Drinfeld's definition [22, Thm. 3.5] suggested to us how to proceed.

Definition 9.4. As in [22, (5.1)], let $H_{r, 1, n}^{*}$ be the algebra generated by the basis $v_{1}, \ldots, v_{n}$ of $V$ and all $\bar{g}(g \in G(r, 1, n))$ such that $\mathbb{C} G(r, 1, n)$ and $S(V)$ are subalgebras and the following additional relations hold:

$$
\begin{array}{rlrl}
\bar{\xi}_{i} v_{k}=v_{k} \bar{\xi}_{i} & & (1 \leq i, k \leq n), \\
\bar{s}_{i} v_{k}=v_{k} \bar{s}_{i} & & (k \neq i, i+1), \\
\bar{s}_{i} v_{i+1} & =v_{i} \bar{s}_{i}+\sum_{a=0}^{r-1}\left(\bar{\xi}_{i}\right)^{a}\left(\bar{\xi}_{i+1}\right)^{-a} & & (1 \leq i \leq n-1),
\end{array}
$$

where $s_{i}$ is the simple reflection $(i, i+1)$.

A useful variant of relation (9.7) is $\bar{s}_{i} v_{i}=v_{i+1} \bar{s}_{i}-\sum_{a=0}^{r-1}\left(\bar{\xi}_{i}\right)^{a}\left(\bar{\xi}_{i+1}\right)^{-a}$. In Dezélée's version [7, one may choose any nonzero scalar as the coefficient of the sum in relation (9.7) above, and the proof of the following theorem may be modified accordingly.

Theorem 9.8. Let $A_{r, 1, n}$ be the graded Hecke algebra of Definition 9.2, There is an algebra isomorphism

$$
A_{r, 1, n} \cong H_{r, 1, n}^{*} \text {. }
$$

The remainder of this section is devoted to the proof of this theorem. We first collect some additional relations in $H_{r, 1, n}^{*}$ that are consequences of (9.6) and (9.7).

Lemma 9.9. Let $1 \leq j, k, m \leq n$ with $j<k$. In $H_{r, 1, n}^{*}$, the element $(\overline{j, k}) v_{m}$ is equal to

$$
\left\{\begin{array}{l}
v_{m}(\overline{j, k}), \quad \text { if } m<j \text { or } k<m, \\
v_{m}(\overline{j, k})+\sum_{a=0}^{r-1}\left(\bar{\xi}_{m}\right)^{a}\left(\bar{\xi}_{k}\right)^{-a}(\overline{j, m, k})-\sum_{a=0}^{r-1}\left(\bar{\xi}_{j}\right)^{a}\left(\bar{\xi}_{m}\right)^{-a}(\overline{j, k, m}), \quad \text { if } j<m<k, \\
v_{k}(\overline{j, k})-\sum_{j<i<k} \sum_{a=0}^{r-1}\left(\bar{\xi}_{i}\right)^{a}\left(\bar{\xi}_{k}\right)^{-a}(\overline{j, i, k})-\sum_{a=0}^{r-1}\left(\bar{\xi}_{j}\right)^{a}\left(\bar{\xi}_{k}\right)^{-a}, \quad \text { if } m=j, \\
v_{j}(\overline{j, k})+\sum_{j<i<k} \sum_{a=0}^{r-1}\left(\bar{\xi}_{i}\right)^{a}\left(\bar{\xi}_{j}\right)^{-a}(\overline{k, i, j})+\sum_{a=0}^{r-1}\left(\bar{\xi}_{j}\right)^{a}\left(\bar{\xi}_{k}\right)^{-a}, \quad \text { if } m=k .
\end{array}\right.
$$

Proof. Write $(\overline{j, k})$ as a product of simple reflections:

$$
(\overline{j, k})=\bar{s}_{k-1} \bar{s}_{k-2} \cdots \bar{s}_{j+1} \bar{s}_{j} \bar{s}_{j+1} \cdots \bar{s}_{k-2} \bar{s}_{k-1} .
$$

If $m<j$ or $k<m$, then $(\overline{j, k}) v_{m}=v_{m}(\overline{j, k})$ by (9.6). If $j<m<k$, then application of (9.7) and its variation (given in the text following (9.7)) yields the stated relation.

If $m=j$, we use induction on $k-j$. If $k-j=1$, then the desired relation is the one given in the text following (9.7). Assume the relation given in Lemma 9.9 holds when $k$ is replaced by $k-1$ and $m=j<k-1$. Then 
$(\overline{j, k}) v_{j}=\bar{s}_{k-1}(\overline{j, k-1}) \bar{s}_{k-1} v_{j}=\bar{s}_{k-1}(\overline{j, k-1}) v_{j} \bar{s}_{k-1}$, and application of the induction hypothesis and the variation of (9.7) yields the relation stated in the theorem. The final relation is proved by a similar induction.

We will make a change of generators for $H_{r, 1, n}^{*}$. For each $k(1 \leq k \leq n)$, let

$$
\widetilde{v}_{k}=v_{k}+\frac{1}{2} \sum_{\substack{1 \leq j \leq n \\ j \neq k}} \sum_{a=0}^{r-1}(-1)^{\delta_{j<k}}\left(\bar{\xi}_{k}\right)^{a}\left(\bar{\xi}_{j}\right)^{-a}(\overline{k, j}),
$$

where $\delta_{j<k}=1$ if $j<k$ and 0 otherwise.

Lemma 9.11. The algebra $H_{r, 1, n}^{*}$ is generated by $\widetilde{v}_{k}(1 \leq k \leq n)$ and $\bar{g}(g \in$ $G(r, 1, n))$ and is defined by the relations of $G(r, 1, n)$ together with

$$
\begin{aligned}
\bar{\xi}_{i} \widetilde{v}_{k} & =\widetilde{v}_{k} \bar{\xi}_{i} \quad \text { for all } 1 \leq i, k \leq n \\
\bar{s}_{i} \widetilde{v}_{k} & =\widetilde{v}_{k} \bar{s}_{i} \quad \text { if } k \notin\{i, i+1\} \\
\bar{s}_{i} \widetilde{v}_{i} & =\widetilde{v}_{i+1} \bar{s}_{i} \quad \text { for } 1 \leq i \leq n-1, \\
\widetilde{v}_{m} \widetilde{v}_{k}-\widetilde{v}_{k} \widetilde{v}_{m} & =\frac{1}{4} \sum_{\substack{1 \leq i \leq n \\
i \neq m, k}} \sum_{a, b=0}^{r-1}\left(\bar{\xi}_{m}\right)^{a}\left(\bar{\xi}_{k}\right)^{b}\left(\bar{\xi}_{i}\right)^{-a-b}((\overline{m, k, i})-(\overline{m, i, k})),
\end{aligned}
$$

where the last relation holds for $1 \leq m<k \leq n$.

Proof. In the definition of $\widetilde{v}_{k}$, both summands $\sum \sum(-1)^{\delta_{j<k}}\left(\bar{\xi}_{k}\right)^{a}\left(\bar{\xi}_{j}\right)^{-a}(\overline{k, j})$ and $v_{k}$ are invariant under conjugation by $\bar{\xi}_{i}(1 \leq i \leq n)$ and by $\bar{s}_{i}(k \neq i, i+1)$, so (9.12) and (9.13) hold.

We check (9.14), using (9.7):

$$
\begin{aligned}
& \bar{s}_{i} \widetilde{v}_{i} \bar{s}_{i}=\bar{s}_{i} v_{i} \bar{s}_{i}+\frac{1}{2} \sum_{\substack{1 \leq j \leq n \\
j \neq i}} \sum_{a=0}^{r-1}(-1)^{\delta_{j<i}} \bar{s}_{i}\left(\bar{\xi}_{i}\right)^{a}\left(\bar{\xi}_{j}\right)^{-a}(\overline{i, j}) \bar{s}_{i} \\
& =v_{i+1}-\sum_{a=0}^{r-1} \bar{s}_{i}\left(\bar{\xi}_{i}\right)^{a}\left(\bar{\xi}_{i+1}\right)^{-a}+\frac{1}{2} \sum_{\substack{1 \leq j \leq n \\
j \neq i+1}} \sum_{a=0}^{r-1}(-1)^{\delta_{j<i}}\left(\bar{\xi}_{i+1}\right)^{a}\left(\bar{\xi}_{j}\right)^{-a}(\overline{i+1, j}) \\
& =v_{i+1}+\frac{1}{2} \sum_{\substack{1 \leq j \leq n \\
j \neq i+1}} \sum_{a=0}^{r-1}(-1)^{\delta_{j<(i+1)}}\left(\bar{\xi}_{i+1}\right)^{a}\left(\bar{\xi}_{j}\right)^{-a}(\overline{i+1, j}) \\
& =\widetilde{v}_{i+1} \text {. }
\end{aligned}
$$

Finally, we check (9.15). Assume $m<k$. We compute the bracket $\widetilde{v}_{m} \widetilde{v}_{k}-\widetilde{v}_{k} \widetilde{v}_{m}$, first substituting from (9.10) and then applying the relations from Lemma 9.9, The 
result is a cancellation of all terms having factors of $v_{m}$ or $v_{k}$ :

$$
\begin{aligned}
& \left(v_{m}+\frac{1}{2} \sum_{\substack{1 \leq i \leq n \\
i \neq m}} \sum_{b=0}^{r-1}(-1)^{\delta_{i<m}}\left(\bar{\xi}_{m}\right)^{b}\left(\bar{\xi}_{i}\right)^{-b}(\overline{m, i})\right) \\
& \times\left(v_{k}+\frac{1}{2} \sum_{\substack{1 \leq j \leq n \\
j \neq k}} \sum_{a=0}^{r-1}(-1)^{\delta_{j<k}}\left(\bar{\xi}_{k}\right)^{a}\left(\bar{\xi}_{j}\right)^{-a}(\overline{k, j})\right) \\
& -\left(v_{k}+\frac{1}{2} \sum_{\substack{1 \leq j \leq n \\
j \neq k}} \sum_{a=0}^{r-1}(-1)^{\delta_{j<k}}\left(\bar{\xi}_{k}\right)^{a}\left(\bar{\xi}_{j}\right)^{-a}(\overline{k, j})\right) \\
& \times\left(v_{m}+\frac{1}{2} \sum_{\substack{1 \leq i \leq n \\
i \neq m}} \sum_{b=0}^{r-1}(-1)^{\delta_{i<m}}\left(\bar{\xi}_{m}\right)^{b}\left(\bar{\xi}_{i}\right)^{-b}(\overline{m, i})\right) \\
& =\frac{1}{2} \sum_{\substack{1 \leq j \leq n \\
j \neq k}} \sum_{a=0}^{r-1}(-1)^{\delta_{j<k}} v_{m}\left(\bar{\xi}_{k}\right)^{a}\left(\bar{\xi}_{j}\right)^{-a}(\overline{k, j}) \\
& +\frac{1}{2} \sum_{\substack{1 \leq i \leq n \\
i \neq m}} \sum_{b=0}^{r-1}(-1)^{\delta_{i<m}}\left(\bar{\xi}_{m}\right)^{b}\left(\bar{\xi}_{i}\right)^{-b}(\overline{m, i}) v_{k}
\end{aligned}
$$

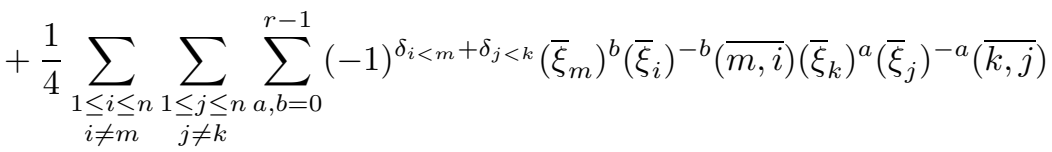

$$
\begin{aligned}
& -\frac{1}{2} \sum_{\substack{1 \leq i \leq n \\
i \neq m}} \sum_{b=0}^{r-1}(-1)^{\delta_{i<m}} v_{k}\left(\bar{\xi}_{m}\right)^{b}\left(\bar{\xi}_{i}\right)^{-b}(\overline{m, i}) \\
& -\frac{1}{2} \sum_{\substack{1 \leq j \leq n \\
j \neq k}} \sum_{a=0}^{r-1}(-1)^{\delta_{j<k}}\left(\bar{\xi}_{k}\right)^{a}\left(\bar{\xi}_{j}\right)^{-a}(\overline{k, j}) v_{m} \\
& -\frac{1}{4} \sum_{\substack{1 \leq i \leq n \\
i \neq m}} \sum_{\substack{1 \leq j \leq n \\
j \neq k}} \sum_{\substack{a, b=0 \\
\text { jon }}}(-1)^{\delta_{i<m}+\delta_{j<k}}\left(\bar{\xi}_{k}\right)^{a}\left(\bar{\xi}_{j}\right)^{-a}(\overline{k, j})\left(\bar{\xi}_{m}\right)^{b}\left(\bar{\xi}_{i}\right)^{-b}(\overline{m, i}) \\
& =\frac{1}{4} \sum_{\substack{1 \leq i \leq n \\
i \neq m}} \sum_{\substack{1 \leq j \leq n \\
j \neq k}} \sum_{a, b=0}^{r-1}(-1)^{\delta_{i<m}+\delta_{j<k}}\left(\bar{\xi}_{m}\right)^{b}\left(\bar{\xi}_{i}\right)^{-b}(\overline{m, i})\left(\bar{\xi}_{k}\right)^{a}\left(\bar{\xi}_{j}\right)^{-a}(\overline{k, j})
\end{aligned}
$$

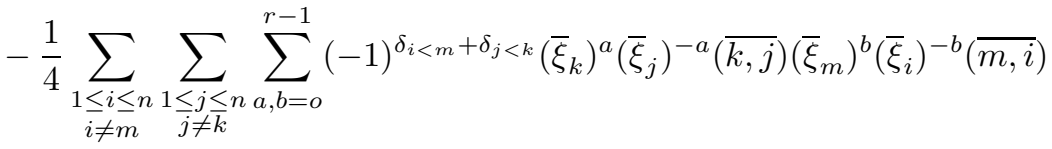

$$
\begin{aligned}
& +\frac{1}{2} \sum_{\substack{1 \leq i \leq n \\
i \neq m, k}} \sum_{a, b=0}^{r-1}\left(\bar{\xi}_{m}\right)^{a}\left(\bar{\xi}_{k}\right)^{b}\left(\bar{\xi}_{i}\right)^{-a-b}((\overline{m, k, i})-(\overline{m, i, k})) .
\end{aligned}
$$


Now in the first two of the three summands above, we may cancel the terms for which $\{m, i\} \cap\{k, j\}$ is empty. Rewriting the remaining terms and combining with the last summand, we have the desired relation (9.15).

Finally, if the $\widetilde{v}_{k}$ are taken instead of the $v_{k}$ as generators of $H_{r, 1, n}^{*}$ together with $G(r, 1, n)$, relations (9.12)-9.15) are equivalent to the relations for $H_{r, 1, n}^{*}$ given in Definition 9.4 .

Proof of Theorem 9.8. Define an algebra homomorphism from $A_{r, 1, n}$ to $H_{r, 1, n}^{*}$ by sending $\bar{g}$ to $\bar{g}(g \in G(r, 1, n))$ and $v_{k}$ to $\frac{2}{\sqrt{3}} \widetilde{v}_{k}(1 \leq k \leq n)$. This map is welldefined: The relations (9.12)-(9.14) correspond to relations in $A_{r, 1, n}$, and (9.15) corresponds to (9.3) in $A_{r, 1, n}$. This accounts for all the relations in $A_{r, 1, n}$. The map is surjective as every generator in $H_{r, 1, n}^{*}$ lies in its image. On the other hand, we can define an inverse map similarly. Therefore $A_{r, 1, n} \cong H_{r, 1, n}^{*}$.

\section{REFERENCES}

[1] S. Ariki and K. Koike, "A Hecke Algebra of $(\mathbb{Z} / r \mathbb{Z}) \prec \mathfrak{S}_{n}$ and Construction of Its Irreducible Representations," Advances in Mathematics, 106 (1994), 216-243. MR1279219 (95h:20006)

[2] M. Broué and G. Malle, "Zyklotomische Heckealgebren", Astérisque, 212 (1993), 119-189. MR:1235834 (94m:20095)

[3] M. Broué, G. Malle, and J. Michel, "Towards Spetses I", Transform. Groups 4 (1999), no. 2-3, 157-218. MR:1712862 (2001b:20082)

[4] M. Broué, G. Malle, and R. Rouquier, "Complex Reflection Groups, Braid Groups, Hecke Algebras," J. reine angew. Math., 500 (1998), 127-190. MR1637497(99m:20088)

[5] A. Căldăraru, A. Giaquinto, and S. Witherspoon, "Algebraic deformations arising from orbifolds with discrete torsion," J. Pure Appl. Algebra 187 (2004), no. 1-3, 51-70. MR2027895 (2005c:16013)

[6] T. Chmutova, "Twisted symplectic reflection algebras," to appear in J. Pure Appl. Algebra.

[7] C. Dezélée, "Une généralisation de l'algèbre de Hecke graduée de type $B, "$ math.RT/0304484.

[8] C. Dezélée, "Generalized graded Hecke algebra for complex reflection group of type $G(r, 1, n), "$ math.RT/0605410.

[9] V. G. Drinfeld, "Degenerate affine Hecke algebras and Yangians," Funct. Anal. Appl. 20 (1986), 58-60. MR831053 (87m:22044)

[10] P. Etingof, "Exploring noncommutative algebras via deformation theory," math. QA/0506144.

[11] P. Etingof and V. Ginzburg, "Symplectic reflection algebras, Calogero-Moser space, and deformed Harish-Chandra homomorphism," Invent. Math. 147 (2002), no. 2, 243-348. MR 1881922 (2003b:16021)

[12] M. Farinati, "Hochschild duality, localization, and smash products," J. Algebra 284 (2005), no. 1, 415-434. MR2115022 (2005j:16009)

[13] M. Gerstenhaber, "On the deformation of rings and algebras," Ann. Math. 79 (1964), 59103. MR.0171807 (30:2034)

[14] V. Ginzburg and D. Kaledin, "Poisson deformations of symplectic quotient singularities," Adv. Math. 186 (2004), no. 1, 1-57. MR2065506 (2005h:32072)

[15] D. Kazhdan and G. Lusztig, "Proof of the Deligne-Langlands conjecture for Hecke algebras," Invent. Math. 87 (1987), no. 1, 153-215. MR862716 (88d:11121)

[16] G. Kemper and H. Derksen, "Computational invariant theory," Invariant Theory and Algebraic Transformation Groups, I. Encyclopaedia of Mathematical Science, 130. SpringerVerlag, Berlin, 2002. MR1918599 (2003g:13004)

[17] G. Lusztig, "Cuspidal local systems and graded Hecke algebras I," Inst. Hautes Études Sci. Publ. Math. 67 (1988), 145-202. MR972345 (90e:22029)

[18] G. Lusztig, "Affine Hecke algebras and their graded version," J. Amer. Math. Soc. 2 (1989), no. 3, 599-635. MR991016 (90e:16049) 
[19] I. G. Macdonald, Symmetric Functions and Hall Polynomials, 2nd ed., Oxford Mathematical Monographs, The Clarendon Press, Oxford University Press, New York, 1995. MR.1354144 (96h:05207)

[20] A. Mathas, "The representation theory of the Ariki-Koike and cyclotomic $q$-Schur algebras," Representation Theory of Algebraic Groups and Quantum Groups, 17-25, Adv. Stud. Pure Math. 40, Math. Soc. Japan, Tokyo, 2004. MR2074597 (2005f:20014)

[21] P. Orlik and H. Terao, Arrangements of Hyperplanes, Grundlehren der Mathematischen Wissenschaften 300, Springer-Verlag, Berlin, 1992. MR.1217488(94e:52014)

[22] A. Ram and A. V. Shepler, "Classification of graded Hecke algebras for complex reflection groups," Comment. Math. Helv. 78 (2003), 308-334. MR1988199 (2004d:20007)

[23] G. C. Shephard and J. A. Todd, "Finite unitary reflection groups," Canad. J. Math. 6 (1954), 274-304. MR0059914 (15:600b)

[24] D. Ştefan, "Hochschild cohomology on Hopf Galois extensions," J. Pure Appl. Algebra 103 (1995), 221-233. MR1358765 (96h:16013)

[25] C. A. Weibel, An Introduction to Homological Algebra, Cambridge Studies in Adv. Math. 38, Cambridge Univ. Press, Cambridge, 1994. MR1269324 (95f:18001)

[26] S. Witherspoon, "Skew derivations and deformations of a family of group crossed products," Commun. Algebra 34 (2006), no. 11, 4187-4206. MR2267580(2007j:16016)

[27] S. Witherspoon, "Twisted graded Hecke algebras," J. Algebra 317 (2007), 30-42.

Department of Mathematics, University of North Texas, Denton, Texas 76203

E-mail address: ashepler@unt.edu

Department of Mathematics, Texas A\&M University, College Station, Texas 77843

E-mail address: sjw@math.tamu.edu 